\title{
Enthalpy of Formation and O-H Bond Dissociation Enthalpy of Phenol: \\ Inconsistency between Theory and Experiment
}

\author{
Olga V. Dorofeeva, Oxana N. Ryzhova \\ Department of Chemistry, Lomonosov Moscow State University, Moscow 119991, Russia
}

Supporting Information 


\section{Content}

page

Table S1. Experimental Enthalpies of Formation of Reference Compounds Used in Isodesmic

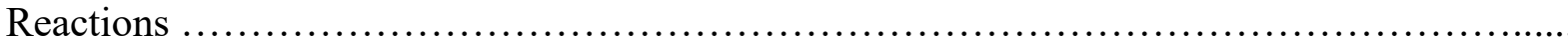

Table S2. Enthalpy of Formation of Gaseous Anisole Calculated from Atomization, Isodesmic, and Isogyric Reactions Using G4 Energies (in $\mathrm{kJ} / \mathrm{mol}$ )

Table S3. Enthalpies of Formation of Gaseous Phenols Calculated from Isodesmic Reactions Using G4 Energies

Table S4. Enthalpies of Formation of Gaseous $\mathrm{C}_{6} \mathrm{H}_{5} \mathrm{O}, \mathrm{C}_{6} \mathrm{H}_{5}$, and $\mathrm{CH}_{3} \mathrm{O}$ Radicals Calculated from Atomization Reaction and Isogyric Reactions Using G4 Energies 
Table S1. Experimental Enthalpies of Formation of Reference Compounds Used in Isodesmic Reactions

\begin{tabular}{|c|c|c|c|c|}
\hline \multicolumn{2}{|l|}{ compound } & $\Delta_{\mathrm{f}} \mathrm{H}_{298}^{\circ}(\mathrm{g}), \mathrm{kJ} / \mathrm{mol}$ & reference & $-\operatorname{calc}(\mathrm{G} 4)^{a}$ \\
\hline \multicolumn{5}{|l|}{ molecules } \\
\hline $\mathrm{CH}_{2} \mathrm{O}$ & $\mathrm{HC}(\mathrm{O}) \mathrm{H}$ & $-109.16 \pm 0.11$ & ATcT: 1 & 2.5 \\
\hline $\mathrm{CH}_{2} \mathrm{O}_{2}$ & $\mathrm{HC}(\mathrm{O}) \mathrm{OH}$ & $-378.51 \pm 0.25$ & ATcT: 1 & 0.5 \\
\hline $\mathrm{CH}_{3} \mathrm{~N}$ & $\mathrm{H}_{2} \mathrm{C}=\mathrm{NH}$ & $88.7 \pm 0.98$ & ATcT: 1 & 1.3 \\
\hline $\mathrm{CH}_{4}$ & $\mathrm{CH}_{4}$ & $-74.520 \pm 0.056$ & ATcT: 2 & 0.2 \\
\hline $\mathrm{CH}_{4} \mathrm{O}$ & $\mathrm{CH}_{3} \mathrm{OH}$ & $-200.71 \pm 0.16$ & ATcT: 2 & 0.5 \\
\hline $\mathrm{CH}_{4} \mathrm{O}_{2}$ & $\mathrm{CH}_{2}(\mathrm{OH})_{2}$ & $-392.59 \pm 0.96$ & ATcT: 1 & 0.0 \\
\hline $\mathrm{CH}_{4} \mathrm{O}_{2}$ & $\mathrm{CH}_{3} \mathrm{OOH}$ & $-127.81 \pm 0.91$ & ATcT: 1 & -0.5 \\
\hline $\mathrm{C}_{2} \mathrm{H}_{4}$ & $\mathrm{H}_{2} \mathrm{C}=\mathrm{CH}_{2}$ & $52.56 \pm 0.15$ & ATcT: 1 & 0.6 \\
\hline $\mathrm{C}_{2} \mathrm{H}_{4} \mathrm{O}$ & $\mathrm{CH}_{3} \mathrm{C}(\mathrm{O}) \mathrm{H}$ & $-165.37 \pm 0.32$ & ATcT: 1 & 1.2 \\
\hline $\mathrm{C}_{2} \mathrm{H}_{6}$ & $\mathrm{CH}_{3} \mathrm{CH}_{3}$ & $-83.91 \pm 0.14$ & ATcT: 2 & -0.4 \\
\hline $\mathrm{C}_{2} \mathrm{H}_{6} \mathrm{O}$ & $\mathrm{CH}_{3} \mathrm{CH}_{2} \mathrm{OH}$ & $-234.57 \pm 0.22$ & ATcT: 1 & -1.4 \\
\hline $\mathrm{C}_{2} \mathrm{H}_{6} \mathrm{O}$ & $\mathrm{CH}_{3} \mathrm{OCH}_{3}$ & $-184.02 \pm 0.44$ & ATcT: 1 & 1.2 \\
\hline $\mathrm{C}_{2} \mathrm{H}_{6} \mathrm{O}_{2}$ & $\mathrm{HOCH}_{2} \mathrm{CH}_{2} \mathrm{OH}$ & $-389.42 \pm 0.49$ & ATcT: 1 & -0.4 \\
\hline $\mathrm{C}_{3} \mathrm{H}_{6}$ & $\mathrm{H}_{2} \mathrm{C}=\mathrm{CHCH}_{3}$ & $20.37 \pm 0.33$ & ATcT: 1 & 0.2 \\
\hline $\mathrm{C}_{3} \mathrm{H}_{6} \mathrm{O}$ & $\mathrm{CH}_{3} \mathrm{C}(\mathrm{O}) \mathrm{CH}_{3}$ & $-216.07 \pm 0.37$ & ATcT: 1 & 0.4 \\
\hline $\mathrm{C}_{3} \mathrm{H}_{8}$ & $\mathrm{CH}_{3} \mathrm{CH}_{2} \mathrm{CH}_{3}$ & $-104.4 \pm 0.3$ & ATcT: 1 & -0.5 \\
\hline $\mathrm{C}_{3} \mathrm{H}_{8} \mathrm{O}$ & $\mathrm{CH}_{3} \mathrm{CH}_{2} \mathrm{CH}_{2} \mathrm{OH}$ & $-255.1 \pm 0.4$ & 3 & -1.6 \\
\hline $\mathrm{C}_{3} \mathrm{H}_{8} \mathrm{O}$ & $\mathrm{CH}_{3} \mathrm{CH}(\mathrm{OH}) \mathrm{CH}_{3}$ & $-272.80 \pm 0.37$ & ATcT: 1 & -0.3 \\
\hline $\mathrm{C}_{3} \mathrm{H}_{8} \mathrm{O}_{2}$ & $\mathrm{HOCH}_{2} \mathrm{CH}_{2} \mathrm{CH}_{2} \mathrm{OH}$ & $-410.6 \pm 2.2$ & 4 & -0.9 \\
\hline $\mathrm{C}_{3} \mathrm{H}_{8} \mathrm{O}_{2}$ & $\mathrm{HOCH}_{2} \mathrm{CH}(\mathrm{OH}) \mathrm{CH}_{3}$ & $-428.4 \pm 1.6$ & 5 & 0.7 \\
\hline $\mathrm{C}_{3} \mathrm{H}_{8} \mathrm{O}_{3}$ & $\mathrm{HOCH}_{2} \mathrm{CH}(\mathrm{OH}) \mathrm{CH}_{2} \mathrm{OH}$ & $-583.3 \pm 3.0$ & estimation, this work ${ }^{b}$ & \\
\hline $\mathrm{C}_{4} \mathrm{H}_{10}$ & $\mathrm{CH}_{3} \mathrm{CH}_{2} \mathrm{CH}_{2} \mathrm{CH}_{3}$ & $-125.85 \pm 0.38$ & ATcT: 1 & -0.9 \\
\hline $\mathrm{C}_{4} \mathrm{H}_{10}$ & $\mathrm{CH}_{3} \mathrm{CH}\left(\mathrm{CH}_{3}\right) \mathrm{CH}_{3}$ & $-135.36 \pm 0.4$ & ATcT: 1 & -1.9 \\
\hline $\mathrm{C}_{4} \mathrm{H}_{10} \mathrm{O}$ & $\mathrm{CH}_{3} \mathrm{CH}_{2} \mathrm{CH}_{2} \mathrm{CH}_{2} \mathrm{OH}$ & $-274.9 \pm 0.4$ & 3 & -0.6 \\
\hline $\mathrm{C}_{4} \mathrm{H}_{10} \mathrm{O}$ & $\mathrm{CH}_{3} \mathrm{CH}_{2} \mathrm{OCH}_{2} \mathrm{CH}_{3}$ & $-252.1 \pm 0.7$ & 3 & 0.7 \\
\hline $\mathrm{C}_{5} \mathrm{H}_{12}$ & $\mathrm{CH}_{3} \mathrm{CH}_{2} \mathrm{CH}_{2} \mathrm{CH}_{2} \mathrm{CH}_{3}$ & $-146.9 \pm 0.8$ & 3 & -0.9 \\
\hline $\mathrm{C}_{6} \mathrm{H}_{5} \mathrm{NO}$ & $\mathrm{C}_{6} \mathrm{H}_{5} \mathrm{NO}$ & $198.6 \pm 1.5$ & ATcT: 6 & 2.5 \\
\hline $\mathrm{C}_{6} \mathrm{H}_{6}$ & $\mathrm{C}_{6} \mathrm{H}_{6}$ & $83.18 \pm 0.26$ & ATcT: 6 & -0.4 \\
\hline $\mathrm{C}_{6} \mathrm{H}_{6} \mathrm{O}$ & $\mathrm{C}_{6} \mathrm{H}_{5} \mathrm{OH}$ & $-91.8 \pm 2.0$ & estimation, this work; see Table 2 & \\
\hline $\mathrm{C}_{6} \mathrm{H}_{6} \mathrm{O}_{2}$ & $1,2-\mathrm{C}_{6} \mathrm{H}_{4}(\mathrm{OH})_{2}$ & $-267.5 \pm 1.9$ & 7 & 2.3 \\
\hline $\mathrm{C}_{6} \mathrm{H}_{6} \mathrm{O}_{2}$ & $1,3-\mathrm{C}_{6} \mathrm{H}_{4}(\mathrm{OH})_{2}$ & -267.7 & estimation, this work; see Table 2 & \\
\hline $\mathrm{C}_{6} \mathrm{H}_{6} \mathrm{O}_{2}$ & $1,4-\mathrm{C}_{6} \mathrm{H}_{4}(\mathrm{OH})_{2}$ & $-260.3 \pm 1.0$ & see Table 2 & -0.4 \\
\hline $\mathrm{C}_{6} \mathrm{H}_{6} \mathrm{O}_{3}$ & $1,2,3-\mathrm{C}_{6} \mathrm{H}_{3}(\mathrm{OH})_{3}$ & $-447.2 \pm 1.0$ & see Table 2 & 0.6 \\
\hline $\mathrm{C}_{6} \mathrm{H}_{6} \mathrm{O}_{3}$ & $1,2,4-\mathrm{C}_{6} \mathrm{H}_{3}(\mathrm{OH})_{3}$ & $-439.6 \pm 1.1$ & see Table 2 & -0.7 \\
\hline $\mathrm{C}_{6} \mathrm{H}_{6} \mathrm{O}_{3}$ & $1,3,5-\mathrm{C}_{6} \mathrm{H}_{3}(\mathrm{OH})_{3}$ & $-444.7 \pm 3.0$ & estimation, this work; see Table 2 & \\
\hline $\mathrm{C}_{7} \mathrm{H}_{6} \mathrm{O}$ & $\mathrm{C}_{6} \mathrm{H}_{5} \mathrm{C}(\mathrm{O}) \mathrm{H}$ & $-36.7 \pm 2.8$ & 3 & 2.0 \\
\hline $\mathrm{C}_{7} \mathrm{H}_{8}$ & $\mathrm{C}_{6} \mathrm{H}_{5} \mathrm{CH}_{3}$ & $50.41 \pm 0.37$ & ATcT: 1 & 0.2 \\
\hline $\mathrm{C}_{7} \mathrm{H}_{8} \mathrm{O}$ & $\mathrm{C}_{6} \mathrm{H}_{5} \mathrm{OCH}_{3}$ & $-70.7 \pm 1.4$ & 8 & 0.6 \\
\hline $\mathrm{C}_{7} \mathrm{H}_{8} \mathrm{O}$ & $1,2-\left(\mathrm{CH}_{3}\right) \mathrm{C}_{6} \mathrm{H}_{4}(\mathrm{OH})$ & $-128.3 \pm 0.9$ & 9 & 0.1 \\
\hline $\mathrm{C}_{7} \mathrm{H}_{8} \mathrm{O}$ & $1,3-\left(\mathrm{CH}_{3}\right) \mathrm{C}_{6} \mathrm{H}_{4}(\mathrm{OH})$ & $-125.6 \pm 3.0$ & estimation, this work; see Table 2 & \\
\hline $\mathrm{C}_{7} \mathrm{H}_{8} \mathrm{O}$ & $1,4-\left(\mathrm{CH}_{3}\right) \mathrm{C}_{6} \mathrm{H}_{4}(\mathrm{OH})$ & $-125.3 \pm 1.5$ & 9 & -1.9 \\
\hline $\mathrm{C}_{8} \mathrm{H}_{8}$ & $\mathrm{C}_{6} \mathrm{H}_{5} \mathrm{CH}=\mathrm{CH}_{2}$ & $148.0 \pm 1.4$ & 3 & 0.4 \\
\hline $\mathrm{C}_{8} \mathrm{H}_{10}$ & $\mathrm{C}_{6} \mathrm{H}_{5} \mathrm{CH}_{2} \mathrm{CH}_{3}$ & $29.9 \pm 0.4$ & 10 & 1.2 \\
\hline $\mathrm{C}_{8} \mathrm{H}_{10}$ & $1,4-\mathrm{C}_{6} \mathrm{H}_{4}\left(\mathrm{CH}_{3}\right)_{2}$ & $18.0 \pm 0.5$ & 11 & 0.1 \\
\hline $\mathrm{C}_{8} \mathrm{H}_{10} \mathrm{O}$ & $2,3-\left(\mathrm{CH}_{3}\right)_{2} \mathrm{C}_{6} \mathrm{H}_{3}(\mathrm{OH})$ & $-157.3 \pm 1.1$ & 12 & 0.9 \\
\hline $\mathrm{C}_{8} \mathrm{H}_{10} \mathrm{O}$ & $2,4-\left(\mathrm{CH}_{3}\right)_{2} \mathrm{C}_{6} \mathrm{H}_{3}(\mathrm{OH})$ & $-159.3 \pm 3.0$ & estimation, this work; see Table 2 & \\
\hline $\mathrm{C}_{8} \mathrm{H}_{10} \mathrm{O}$ & $2,5-\left(\mathrm{CH}_{3}\right)_{2} \mathrm{C}_{6} \mathrm{H}_{3}(\mathrm{OH})$ & $-161.7 \pm 0.5$ & 12 & -0.6 \\
\hline $\mathrm{C}_{8} \mathrm{H}_{10} \mathrm{O}$ & $2,6-\left(\mathrm{CH}_{3}\right)_{2} \mathrm{C}_{6} \mathrm{H}_{3}(\mathrm{OH})$ & $-161.8 \pm 0.5$ & 12 & 1.0 \\
\hline $\mathrm{C}_{8} \mathrm{H}_{10} \mathrm{O}$ & $3,4-\left(\mathrm{CH}_{3}\right)_{2} \mathrm{C}_{6} \mathrm{H}_{3}(\mathrm{OH})$ & $-156.6 \pm 0.6$ & 12 & 1.3 \\
\hline $\mathrm{C}_{8} \mathrm{H}_{10} \mathrm{O}$ & $3,5-\left(\mathrm{CH}_{3}\right)_{2} \mathrm{C}_{6} \mathrm{H}_{3}(\mathrm{OH})$ & $-161.6 \pm 0.7$ & 12 & -2.3 \\
\hline $\mathrm{C}_{9} \mathrm{H}_{12} \mathrm{O}$ & $2,3,6-\left(\mathrm{CH}_{3}\right)_{3} \mathrm{C}_{6} \mathrm{H}_{2}(\mathrm{OH})$ & $-192.9 \pm 1.4$ & 13 & -0.7 \\
\hline $\mathrm{C}_{9} \mathrm{H}_{12} \mathrm{O}$ & $2,4,6-\left(\mathrm{CH}_{3}\right)_{3} \mathrm{C}_{6} \mathrm{H}_{2}(\mathrm{OH})$ & $-192.4 \pm 1.3$ & 13 & 1.5 \\
\hline
\end{tabular}




\begin{tabular}{|c|c|c|c|c|}
\hline $\mathrm{HNO}$ & $\mathrm{HNO}$ & $106.92 \pm 0.11$ & ATcT: 1 & 1.9 \\
\hline $\mathrm{HNO}_{2}$ & $\mathrm{HNO}_{2}$ & $-79.188 \pm 0.079$ & ATcT: 1 & -0.2 \\
\hline $\mathrm{H}_{2} \mathrm{O}$ & $\mathrm{H}_{2} \mathrm{O}$ & $-241.834 \pm 0.026$ & ATcT: 2 & -1.7 \\
\hline $\mathrm{H}_{2} \mathrm{O}_{2}$ & $\mathrm{HOOH}$ & $-135.442 \pm 0.064$ & ATcT: 1 & -2.9 \\
\hline \multicolumn{5}{|l|}{ radicals } \\
\hline $\mathrm{CHO}$ & $\mathrm{HCO} \bullet$ & $41.80 \pm 0.10$ & ATcT: 2 & 3.0 \\
\hline $\mathrm{CH}_{3}$ & $\mathrm{CH}_{3} \bullet$ & $146.374 \pm 0.080$ & ATcT: 2 & 2.1 \\
\hline $\mathrm{CH}_{3} \mathrm{O}$ & $\mathrm{CH}_{3} \mathrm{O} \bullet$ & $21.53 \pm 0.34$ & ATcT: 2 & 4.4 \\
\hline $\mathrm{CH}_{3} \mathrm{O}$ & $\mathrm{HOCH}_{2} \bullet$ & $-16.57 \pm 0.33$ & ATcT: 2 & 1.6 \\
\hline $\mathrm{C}_{2} \mathrm{H}_{3}$ & $\mathrm{H}_{2} \mathrm{C}=\mathrm{CH} \bullet$ & $297.27 \pm 0.47$ & ATcT: 1 & 3.3 \\
\hline $\mathrm{C}_{2} \mathrm{H}_{3} \mathrm{O}$ & $\mathrm{CH}_{3} \mathrm{CO} \bullet$ & $-9.68 \pm 0.4$ & ATcT: 1 & 3.6 \\
\hline $\mathrm{C}_{2} \mathrm{H}_{5}$ & $\mathrm{CH}_{3} \mathrm{CH}_{2} \bullet$ & $119.86 \pm 0.28$ & ATcT: 2 & 0.2 \\
\hline $\mathrm{C}_{2} \mathrm{H}_{5} \mathrm{O}$ & $\mathrm{CH}_{3} \mathrm{CH}_{2} \mathrm{O}$ & $-11.95 \pm 0.5$ & ATcT: 1 & 4.4 \\
\hline $\mathrm{C}_{2} \mathrm{H}_{5} \mathrm{O}$ & $\mathrm{HOCH}_{2} \mathrm{CH}_{2} \bullet$ & $-25.82 \pm 0.61$ & ATcT: 1 & 2.5 \\
\hline $\mathrm{C}_{6} \mathrm{H}_{5}$ & $\mathrm{C}_{6} \mathrm{H}_{5} \bullet$ & $337.3 \pm 0.6$ & ATcT: 6 & 0.6 \\
\hline $\mathrm{C}_{6} \mathrm{H}_{5} \mathrm{O}$ & $\mathrm{C}_{6} \mathrm{H}_{5} \mathrm{O} \cdot$ & $59.6 \pm 2.5$ & estimation, this work ${ }^{c}$ & 2.2 \\
\hline $\mathrm{H}$ & $\mathrm{H} \bullet$ & $217.998 \pm 0.000$ & ATcT: 1 & 0.0 \\
\hline $\mathrm{HO}$ & $\mathrm{HO} \bullet$ & $37.490 \pm 0.026$ & ATcT: 2 & 1.3 \\
\hline $\mathrm{HO}_{2}$ & $\mathrm{HOO} \bullet$ & $12.3 \pm 0.5$ & ATcT: 1 & -0.2 \\
\hline NO & •NO & $91.089 \pm 0.064$ & ATcT: 6 & 2.1 \\
\hline \multicolumn{5}{|l|}{ ions } \\
\hline $\mathrm{CH}_{3}{ }^{+}$ & $\mathrm{CH}_{3}{ }^{+}$ & $1095.517 \pm 0.078$ & ATcT: 1 & -0.1 \\
\hline $\mathrm{CH}_{3} \mathrm{O}^{+}$ & $\mathrm{CH}_{2} \mathrm{OH}^{+}$ & $710.06 \pm 0.24$ & ATcT: 1 & -0.8 \\
\hline $\mathrm{CH}_{3} \mathrm{O}^{+}$ & ${ }^{3} \mathrm{CH}_{3} \mathrm{O}^{+}$ & $1054.98 \pm 0.71$ & ATcT: 1 & 2.7 \\
\hline $\mathrm{C}_{2} \mathrm{H}_{5}^{+}$ & $\mathrm{CH}_{3} \mathrm{CH}_{2}^{+}$ & $902.9 \pm 0.39$ & ATcT: 1 & -4.6 \\
\hline $\mathrm{C}_{6} \mathrm{H}_{5}^{+}$ & $\mathrm{C}_{6} \mathrm{H}_{5}^{+}$ & $1136.84 \pm 0.95$ & ATcT: 6 & 0.6 \\
\hline $\mathrm{C}_{6} \mathrm{H}_{5}^{-}$ & $\mathrm{C}_{6} \mathrm{H}_{5}^{-}$ & $231.99 \pm 0.44$ & ATcT: 6 & 6.7 \\
\hline $\mathrm{HO}^{-}$ & $\mathrm{HO}^{-}$ & $-139.047 \pm 0.027$ & ATcT: 1 & 5.6 \\
\hline
\end{tabular}

${ }^{a}$ Difference between the ATcT or experimental value and calculated in this work by G4 method using atomization reaction. ${ }^{b}$ This value for glycerol was estimated by $\mathrm{G} 4$ method using 21 isodesmic reactions with diols $\left[\mathrm{CH}_{2}(\mathrm{OH})_{2}, \mathrm{HOCH}_{2} \mathrm{CH}_{2} \mathrm{OH}\right.$, $\left.\mathrm{HOCH}_{2} \mathrm{CH}_{2} \mathrm{CH}_{2} \mathrm{OH}, \mathrm{HOCH}_{2} \mathrm{CH}(\mathrm{OH}) \mathrm{CH}_{3}\right]$, alcohols $\left[\mathrm{CH}_{3} \mathrm{OH}, \mathrm{CH}_{3} \mathrm{CH}_{2} \mathrm{OH}, \mathrm{CH}_{3} \mathrm{CH}_{2} \mathrm{CH}_{2} \mathrm{OH}, \mathrm{CH}_{3} \mathrm{CH}(\mathrm{OH}) \mathrm{CH}_{3}\right]$, and aromatic diols and triols (1,2- and 1,4-dihydroxybenzene, 1,2,3- and 1,2,4-trihydroxybenzene). The calculated value is consistent with the experimental enthalpies of formation of the above compounds, however, it is different from the experimental value of $-578.8 \pm 0.6 \mathrm{~kJ} / \mathrm{mol}$, ref. 14 . ${ }^{c}$ See Table S4 of Supporting Information.

\section{References}

(1) Ruscic, B. Updated Active Thermochemical Tables (ATcT) values based on ver. 1.112 of the Thermochemical Network (2012); available at ATcT.anl.gov; last update 27 May 2014.

(2) Ruscic, B. Active Thermochemical Tables: Sequential Bond Dissociation Enthalpies of Methane, Ethane, and Methanol and the Related Thermochemistry. J. Phys. Chem. A 2015, 119, 7810-7837.

(3) Pedley, J. B. Thermochemical Data and Structures of Organic Compounds; Thermodynamics Research Center: College Station, TX, 1994; Vol. I.

(4) Emel'yanenko, V. N.; Verevkin, S. P. Benchmark Thermodynamic Properties of 1,3-Propanediol: Comprehensive Experimental and Theoretical Study. J. Chem. Thermodyn. 2015, 85, 111-119.

(5) Verevkin, S. P.; Emel'yanenko, V. N.; Nell, G. 1,2-Propanediol. Comprehensive Experimental and Theoretical Study. J. Chem. Thermodyn. 2009, 41, 1125-1131.

(6) Stevens, W. R.; Ruscic, B.; Baer, T. Heats of Formation of $\mathrm{C}_{6} \mathrm{H}_{5}{ }^{\bullet}, \mathrm{C}_{6} \mathrm{H}_{5}{ }^{+}$, and $\mathrm{C}_{6} \mathrm{H}_{5} \mathrm{NO}$ by Threshold Photoelectron Photoion Coincidence and Active Thermochemical Tables Analysis. J. Phys. Chem. A 2010, 114, 13134-13145.

(7) Ribeiro da Silva, M. D. M. C.; Ribeiro da Silva, M. A. V.; Pilcher, G. Enthalpies of combustion 1,2-dihydroxybenzene and of six alkylsubstituted 1,2-dihydroxybenzenes. J. Chem. Thermodyn. 1984, 16, 1149-1155.

(8) Simões, R. G.; Agapito, F.; Diogo, H. P.; Minas da Piedade M. E. Enthalpy of Formation of Anisole: Implications for the Controversy on the O-H Bond Dissociation Enthalpy in Phenol. J. Phys. Chem. A 2014, 118, 11026-11032.

(9) Cox, J. D.; The Heats of Combustion of Phenol and the Three Cresols. Pure Appl. Chem. 1961, 2, 125-128.

(10) Chirico, R. D.; Knipmeyer, S. E.; Nguyen, A.; Steele, W. V. Thermodynamic Equilibria in Xylene Isomerization. 4. The Thermodynamic Properties of Ethylbenzene. J. Chem. Eng. Data 1997, 42, 772-783. 
(11) Chirico, R. D.; Knipmeyer, S. E.; Nguyen, A.; Steele, W. V. Thermodynamic Equilibria in Xylene Isomerization. 1. The Thermodynamic Properties of $p$-Xylene. J. Chem. Eng. Data 1997, 42, 248-261.

(12) Andon, R. J. L.; Biddiscombe, D. P.; Cox, J. D.; Handley, R.; Harrop, D.; Herington, E. F. G.; Martin, J. F. Thermodynamic Properties of Organic Oxygen Compounds. Part I. Preparation and Physical Properties of Pure Phenol, Cresols, and Xylenols. J. Chem. Soc. 1960, 5246-5254.

(13) Verevkin, S. P. Thermochemistry of Phenols: Buttress Effects in Sterically Hindered Phenols. J. Chem. Thermodyn. 1999, 31, 1397-1416.

(14) Verevkin, S. P.; Zaitsau, D. H.; Emel'yanenko, V. N.; Zhabina, A. A. Thermodynamic Properties of Glycerol: Experimental and Theoretical Study. Fluid Phase Equilib. 2015, 397, 87-94. 
Table S2. Enthalpy of Formation of Anisole Calculated from Atomization, Isodesmic, and Isogyric Reactions Using G4 Energies (in kJ/mol)

\begin{tabular}{|c|c|c|c|}
\hline \multicolumn{2}{|c|}{ reaction $^{a}$} & $\Delta_{\mathrm{r}} H_{298}^{\circ}$ & $\Delta_{\mathrm{f}} H_{298}^{\circ}{ }^{\circ}$ \\
\hline \multicolumn{4}{|c|}{ atomization reaction } \\
\hline & $\mathrm{C}_{6} \mathrm{H}_{5} \mathrm{OCH}_{3} \rightarrow 7 \mathrm{C}(\mathrm{cr})+4 \mathrm{H}_{2}(\mathrm{~g})+1 / 2 \mathrm{O}_{2}(\mathrm{~g})$ & & -69.8 \\
\hline \multicolumn{4}{|c|}{ isodesmic reactions } \\
\hline 1 & $\mathrm{C}_{6} \mathrm{H}_{5} \mathrm{OCH}_{3}+\mathrm{CH}_{4} \rightarrow \mathrm{C}_{6} \mathrm{H}_{6}+\mathrm{CH}_{3} \mathrm{OCH}_{3}$ & 44.4 & -71.0 \\
\hline 2 & $\mathrm{C}_{6} \mathrm{H}_{5} \mathrm{OCH}_{3}+\mathrm{CH}_{4} \rightarrow \mathrm{C}_{6} \mathrm{H}_{6}+\mathrm{CH}_{3} \mathrm{CH}_{2} \mathrm{OH}$ & -3.8 & -73.4 \\
\hline 3 & $\mathrm{C}_{6} \mathrm{H}_{5} \mathrm{OCH}_{3}+\mathrm{CH}_{3} \mathrm{CH}_{3} \rightarrow \mathrm{C}_{6} \mathrm{H}_{6}+\mathrm{CH}_{3} \mathrm{CH}(\mathrm{OH}) \mathrm{CH}_{3}$ & -34.1 & -71.9 \\
\hline 4 & $\mathrm{C}_{6} \mathrm{H}_{5} \mathrm{OCH}_{3}+\mathrm{CH}_{3} \mathrm{CH}_{2} \mathrm{CH}_{3} \rightarrow \mathrm{C}_{6} \mathrm{H}_{6}+\mathrm{CH}_{3} \mathrm{CH}_{2} \mathrm{OCH}_{2} \mathrm{CH}_{3}$ & 6.0 & -70.9 \\
\hline 5 & $\mathrm{C}_{6} \mathrm{H}_{5} \mathrm{OCH}_{3}+\mathrm{H}_{2} \mathrm{O} \rightarrow \mathrm{C}_{6} \mathrm{H}_{6}+\mathrm{CH}_{2}(\mathrm{OH})_{2}$ & 2.4 & -70.3 \\
\hline 6 & $\mathrm{C}_{6} \mathrm{H}_{5} \mathrm{OCH}_{3}+\mathrm{CH}_{3} \mathrm{OH} \rightarrow \mathrm{C}_{6} \mathrm{H}_{6}+\mathrm{HOCH}_{2} \mathrm{CH}_{2} \mathrm{OH}$ & -33.0 & -72.8 \\
\hline 7 & $\mathrm{C}_{6} \mathrm{H}_{5} \mathrm{OCH}_{3}+\mathrm{H}_{2} \mathrm{O} \rightarrow \mathrm{C}_{6} \mathrm{H}_{6}+\mathrm{CH}_{3} \mathrm{OOH}$ & 267.7 & -70.8 \\
\hline 8 & $\mathrm{C}_{6} \mathrm{H}_{5} \mathrm{OCH}_{3}+\mathrm{CH}_{4} \rightarrow \mathrm{C}_{6} \mathrm{H}_{5} \mathrm{CH}_{3}+\mathrm{CH}_{3} \mathrm{OH}$ & -5.0 & -70.8 \\
\hline 9 & $\mathrm{C}_{6} \mathrm{H}_{5} \mathrm{OCH}_{3}+\mathrm{CH}_{3} \mathrm{CH}_{3} \rightarrow \mathrm{C}_{6} \mathrm{H}_{5} \mathrm{CH}_{3}+\mathrm{CH}_{3} \mathrm{OCH}_{3}$ & 19.7 & -69.4 \\
\hline 10 & $\mathrm{C}_{6} \mathrm{H}_{5} \mathrm{OCH}_{3}+\mathrm{CH}_{3} \mathrm{CH}_{3} \rightarrow \mathrm{C}_{6} \mathrm{H}_{5} \mathrm{CH}_{3}+\mathrm{CH}_{3} \mathrm{CH}_{2} \mathrm{OH}$ & -28.5 & -71.8 \\
\hline 11 & $\mathrm{C}_{6} \mathrm{H}_{5} \mathrm{OCH}_{3}+\mathrm{CH}_{3} \mathrm{CH}_{2} \mathrm{CH}_{3} \rightarrow \mathrm{C}_{6} \mathrm{H}_{5} \mathrm{CH}_{3}+\mathrm{CH}_{3} \mathrm{CH}(\mathrm{OH}) \mathrm{CH}_{3}$ & -47.1 & -70.9 \\
\hline 12 & $\mathrm{C}_{6} \mathrm{H}_{5} \mathrm{OCH}_{3}+\mathrm{CH}_{3} \mathrm{OH} \rightarrow \mathrm{C}_{6} \mathrm{H}_{5} \mathrm{CH}_{3}+\mathrm{CH}_{2}(\mathrm{OH})_{2}$ & -69.9 & -71.6 \\
\hline 13 & $\mathrm{C}_{6} \mathrm{H}_{5} \mathrm{OCH}_{3}+\mathrm{CH}_{3} \mathrm{CH}_{2} \mathrm{OH} \rightarrow \mathrm{C}_{6} \mathrm{H}_{5} \mathrm{CH}_{3}+\mathrm{HOCH}_{2} \mathrm{CH}_{2} \mathrm{OH}$ & -34.1 & -70.3 \\
\hline 14 & $\mathrm{C}_{6} \mathrm{H}_{5} \mathrm{OCH}_{3}+\mathrm{CH}_{3} \mathrm{OH} \rightarrow \mathrm{C}_{6} \mathrm{H}_{5} \mathrm{CH}_{3}+\mathrm{CH}_{3} \mathrm{OOH}$ & 195.4 & -72.1 \\
\hline 15 & $\mathrm{C}_{6} \mathrm{H}_{5} \mathrm{OCH}_{3}+\mathrm{HNO} \rightarrow \mathrm{C}_{6} \mathrm{H}_{5} \mathrm{NO}+\mathrm{CH}_{3} \mathrm{OH}$ & -38.8 & -70.2 \\
\hline 16 & $\mathrm{C}_{6} \mathrm{H}_{5} \mathrm{OCH}_{3}+\mathrm{CH}_{3} \mathrm{CH}_{3} \rightarrow \mathrm{C}_{6} \mathrm{H}_{6}+\mathrm{CH}_{3} \mathrm{CH}_{2} \mathrm{CH}_{2} \mathrm{OH}$ & -15.1 & -73.2 \\
\hline 17 & $\mathrm{C}_{6} \mathrm{H}_{5} \mathrm{OCH}_{3}+\mathrm{CH}_{3} \mathrm{CH}_{2} \mathrm{CH}_{3} \rightarrow \mathrm{C}_{6} \mathrm{H}_{5} \mathrm{CH}_{3}+\mathrm{CH}_{3} \mathrm{CH}_{2} \mathrm{CH}_{2} \mathrm{OH}$ & -28.1 & -72.2 \\
\hline 18 & $\mathrm{C}_{6} \mathrm{H}_{5} \mathrm{OCH}_{3}+\mathrm{CH}_{3} \mathrm{CH}_{2} \mathrm{CH}_{2} \mathrm{CH}_{3} \rightarrow \mathrm{C}_{6} \mathrm{H}_{5} \mathrm{CH}_{3}+\mathrm{CH}_{3} \mathrm{CH}_{2} \mathrm{CH}_{2} \mathrm{CH}_{2} \mathrm{OH}$ & -27.8 & -70.8 \\
\hline 19 & $\mathrm{C}_{6} \mathrm{H}_{5} \mathrm{OCH}_{3}+\mathrm{CH}_{3} \mathrm{CH}_{2} \mathrm{CH}_{2} \mathrm{CH}_{3} \rightarrow \mathrm{C}_{6} \mathrm{H}_{5} \mathrm{CH}_{3}+\mathrm{CH}_{3} \mathrm{CH}_{2} \mathrm{OCH}_{2} \mathrm{CH}_{3}$ & -6.4 & -69.6 \\
\hline \multirow[t]{2}{*}{20} & $\mathrm{C}_{6} \mathrm{H}_{5} \mathrm{OCH}_{3}+\mathrm{CH}_{3} \mathrm{C}(\mathrm{O}) \mathrm{H} \rightarrow \mathrm{C}_{6} \mathrm{H}_{5} \mathrm{C}(\mathrm{O}) \mathrm{H}+\mathrm{CH}_{3} \mathrm{OCH}_{3}$ & 13.8 & -69.1 \\
\hline & average of isodesmi & reactions 1-20: & -71.1 \\
\hline \multicolumn{4}{|c|}{ isogyric reactions } \\
\hline 21 & $\mathrm{C}_{6} \mathrm{H}_{5} \mathrm{OCH}_{3}+\mathrm{H} \bullet \rightarrow \mathrm{C}_{6} \mathrm{H}_{5} \bullet+\mathrm{CH}_{3} \mathrm{OH}$ & -11.3 & -70.1 \\
\hline 22 & $\mathrm{C}_{6} \mathrm{H}_{5} \mathrm{OCH}_{3}+\mathrm{H} \bullet \rightarrow \mathrm{C}_{6} \mathrm{H}_{5} \mathrm{CH}_{3}+\mathrm{HO} \bullet$ & -60.4 & -69.7 \\
\hline 23 & $\mathrm{C}_{6} \mathrm{H}_{5} \mathrm{OCH}_{3}+\mathrm{CH}_{3} \bullet \rightarrow \mathrm{C}_{6} \mathrm{H}_{5} \bullet+\mathrm{CH}_{3} \mathrm{OCH}_{3}$ & 78.3 & -71.5 \\
\hline 24 & $\mathrm{C}_{6} \mathrm{H}_{5} \mathrm{OCH}_{3}+\mathrm{CH}_{3} \cdot \rightarrow \mathrm{C}_{6} \mathrm{H}_{5} \mathrm{CH}_{2} \mathrm{CH}_{3}+\mathrm{HO} \bullet$ & -8.9 & -70.2 \\
\hline 25 & $\mathrm{C}_{6} \mathrm{H}_{5} \mathrm{OCH}_{3}+\mathrm{CH}_{3} \bullet \rightarrow \mathrm{C}_{6} \mathrm{H}_{5} \mathrm{CH}_{3}+\mathrm{CH}_{3} \mathrm{O} \bullet$ & -5.5 & -68.7 \\
\hline 26 & $\mathrm{C}_{6} \mathrm{H}_{5} \mathrm{OCH}_{3}+\mathrm{CH}_{3} \bullet \rightarrow \mathrm{C}_{6} \mathrm{H}_{5} \mathrm{CH}_{3}+\mathrm{HOCH}_{2} \bullet$ & -40.7 & -71.5 \\
\hline 27 & $\mathrm{C}_{6} \mathrm{H}_{5} \mathrm{OCH}_{3}+\bullet \mathrm{NO} \rightarrow \mathrm{C}_{6} \mathrm{H}_{5} \mathrm{NO}+\mathrm{HOCH}_{2} \bullet$ & 160.8 & -69.3 \\
\hline 28 & $\mathrm{C}_{6} \mathrm{H}_{5} \mathrm{OCH}_{3}+\mathrm{CH}_{3} \mathrm{CH}_{2} \bullet \rightarrow \mathrm{C}_{6} \mathrm{H}_{5} \mathrm{CH}_{3}+\mathrm{HOCH}_{2} \mathrm{CH}_{2} \bullet$ & -26.6 & -68.7 \\
\hline 29 & $\mathrm{C}_{6} \mathrm{H}_{5} \mathrm{OCH}_{3}+\mathrm{CH}_{3} \bullet \rightarrow \mathrm{C}_{6} \mathrm{H}_{5} \mathrm{O} \bullet+\mathrm{CH}_{3} \mathrm{CH}_{3}$ & -99.3 & -71.3 \\
\hline 30 & $\mathrm{C}_{6} \mathrm{H}_{5} \mathrm{OCH}_{3}+\mathrm{CH}_{3}^{+} \rightarrow \mathrm{C}_{6} \mathrm{H}_{5}^{+}+\mathrm{CH}_{3} \mathrm{OCH}_{3}$ & -72.7 & -70.0 \\
\hline 31 & $\mathrm{C}_{6} \mathrm{H}_{5} \mathrm{OCH}_{3}+\mathrm{HO}^{-} \rightarrow \mathrm{C}_{6} \mathrm{H}_{5}^{-}+\mathrm{CH}_{3} \mathrm{OOH}$ & 314.1 & -70.8 \\
\hline 32 & $\mathrm{C}_{6} \mathrm{H}_{5} \mathrm{OCH}_{3}+\mathrm{CH}_{3}^{+} \rightarrow \mathrm{C}_{6} \mathrm{H}_{5} \mathrm{CH}_{3}+\mathrm{CH}_{2} \mathrm{OH}^{+}$ & -263.7 & -71.6 \\
\hline 33 & $\mathrm{C}_{6} \mathrm{H}_{5} \mathrm{OCH}_{3}+\mathrm{CH}_{3}^{+} \rightarrow \mathrm{C}_{6} \mathrm{H}_{5} \mathrm{CH}_{3}+{ }^{3} \mathrm{CH}_{3} \mathrm{O}^{+}$ & 78.9 & -69.3 \\
\hline \multirow[t]{4}{*}{34} & $\mathrm{C}_{6} \mathrm{H}_{5} \mathrm{OCH}_{3}+\mathrm{CH}_{3} \mathrm{CH}_{2}^{+} \rightarrow \mathrm{C}_{6} \mathrm{H}_{5}^{+}+\mathrm{CH}_{3} \mathrm{CH}_{2} \mathrm{CH}_{2} \mathrm{OH}$ & 48.5 & -69.7 \\
\hline & average of isogyric & eactions 21-34: & -70.2 \\
\hline & averag & of 34 reactions: & $-70.7 \pm 2.5$ \\
\hline & & experiment: & $-70.7 \pm 1.4^{c}$ \\
\hline
\end{tabular}

${ }^{a}$ Experimental enthalpies of formation of all species used in isodesmic reactions are given in Table S1. These values were taken from the ATcT except for the blue marked species. ${ }^{b}$ The values of enthalpies of reaction $\left(\Delta_{\mathrm{r}} H_{298}^{\circ}\right)$ are given together with enthalpies of formation $\left(\Delta_{\mathrm{f}} H_{298}^{\circ}\right.$ ) for each isodesmic reaction. ${ }^{c}$ Reference 8 in Table S1. 
Table S3. Enthalpies of Formation of Gaseous Phenols Calculated from Isodesmic Reactions Using G4 Energies

\begin{tabular}{|c|c|c|c|}
\hline \multicolumn{2}{|c|}{ reaction $^{a}$} & \multirow{2}{*}{$\begin{array}{l}\Delta_{\mathrm{r}} H_{298}^{\circ}{ }^{b} \\
\mathrm{~kJ} / \mathrm{mol}\end{array}$} & \multirow{2}{*}{$\begin{array}{l}\Delta_{\mathrm{f}} H_{298}^{\circ} \\
\mathrm{kJ} / \mathrm{mol}\end{array}$} \\
\hline \multicolumn{2}{|r|}{ 2-methylphenol } & & \\
\hline 1 & $1,2-\left(\mathrm{CH}_{3}\right) \mathrm{C}_{6} \mathrm{H}_{4}(\mathrm{OH})+\mathrm{CH}_{4} \rightarrow \mathrm{C}_{6} \mathrm{H}_{5} \mathrm{CH}_{3}+\mathrm{CH}_{3} \mathrm{OH}$ & 52.1 & -128.2 \\
\hline 2 & $1,2-\left(\mathrm{CH}_{3}\right) \mathrm{C}_{6} \mathrm{H}_{4}(\mathrm{OH})+\mathrm{CH}_{4} \rightarrow \mathrm{C}_{6} \mathrm{H}_{6}+\mathrm{CH}_{3} \mathrm{OCH}_{3}$ & 101.5 & -128.1 \\
\hline 3 & $1,2-\left(\mathrm{CH}_{3}\right) \mathrm{C}_{6} \mathrm{H}_{4}(\mathrm{OH})+\mathrm{CH}_{3} \mathrm{CH}_{3} \rightarrow \mathrm{C}_{6} \mathrm{H}_{5} \mathrm{OH}+\mathrm{CH}_{3} \mathrm{CH}_{2} \mathrm{CH}_{3}$ & 16.0 & -128.3 \\
\hline 4 & $1,2-\left(\mathrm{CH}_{3}\right) \mathrm{C}_{6} \mathrm{H}_{4}(\mathrm{OH}) \rightarrow \mathrm{C}_{6} \mathrm{H}_{5} \mathrm{OCH}_{3}$ & 57.2 & -127.9 \\
\hline & & average: & $-128.1 \pm 3.0$ \\
\hline \multicolumn{4}{|c|}{ 3-methylphenol } \\
\hline 1 & $1,3-\left(\mathrm{CH}_{3}\right) \mathrm{C}_{6} \mathrm{H}_{4}(\mathrm{OH})+\mathrm{CH}_{4} \rightarrow \mathrm{C}_{6} \mathrm{H}_{5} \mathrm{CH}_{3}+\mathrm{CH}_{3} \mathrm{OH}$ & 49.5 & -125.6 \\
\hline 2 & $1,3-\left(\mathrm{CH}_{3}\right) \mathrm{C}_{6} \mathrm{H}_{4}(\mathrm{OH})+\mathrm{CH}_{4} \rightarrow \mathrm{C}_{6} \mathrm{H}_{6}+\mathrm{CH}_{3} \mathrm{OCH}_{3}$ & 98.9 & -125.5 \\
\hline 3 & $1,3-\left(\mathrm{CH}_{3}\right) \mathrm{C}_{6} \mathrm{H}_{4}(\mathrm{OH})+\mathrm{CH}_{3} \mathrm{CH}_{3} \rightarrow \mathrm{C}_{6} \mathrm{H}_{5} \mathrm{OH}+\mathrm{CH}_{3} \mathrm{CH}_{2} \mathrm{CH}_{3}$ & 13.4 & -125.7 \\
\hline 4 & $1,3-\left(\mathrm{CH}_{3}\right) \mathrm{C}_{6} \mathrm{H}_{4}(\mathrm{OH}) \rightarrow \mathrm{C}_{6} \mathrm{H}_{5} \mathrm{OCH}_{3}$ & 54.5 & -125.2 \\
\hline 5 & $1,3-\left(\mathrm{CH}_{3}\right) \mathrm{C}_{6} \mathrm{H}_{4}(\mathrm{OH}) \rightarrow 1,2-\left(\mathrm{CH}_{3}\right) \mathrm{C}_{6} \mathrm{H}_{4}(\mathrm{OH})$ & -2.6 & -126.0 \\
\hline & & average: & $-125.6 \pm 3.0$ \\
\hline \multicolumn{4}{|c|}{ 4-methylphenol } \\
\hline 1 & $1,4-\left(\mathrm{CH}_{3}\right) \mathrm{C}_{6} \mathrm{H}_{4}(\mathrm{OH})+\mathrm{CH}_{4} \rightarrow \mathrm{C}_{6} \mathrm{H}_{5} \mathrm{CH}_{3}+\mathrm{CH}_{3} \mathrm{OH}$ & 47.1 & -123.2 \\
\hline 2 & $1,4-\left(\mathrm{CH}_{3}\right) \mathrm{C}_{6} \mathrm{H}_{4}(\mathrm{OH})+\mathrm{CH}_{4} \rightarrow \mathrm{C}_{6} \mathrm{H}_{6}+\mathrm{CH}_{3} \mathrm{OCH}_{3}$ & 96.5 & -123.1 \\
\hline 3 & $1,4-\left(\mathrm{CH}_{3}\right) \mathrm{C}_{6} \mathrm{H}_{4}(\mathrm{OH})+\mathrm{CH}_{3} \mathrm{CH}_{3} \rightarrow \mathrm{C}_{6} \mathrm{H}_{5} \mathrm{OH}+\mathrm{CH}_{3} \mathrm{CH}_{2} \mathrm{CH}_{3}$ & 11.0 & -123.3 \\
\hline 4 & $1,4-\left(\mathrm{CH}_{3}\right) \mathrm{C}_{6} \mathrm{H}_{4}(\mathrm{OH}) \rightarrow \mathrm{C}_{6} \mathrm{H}_{5} \mathrm{OCH}_{3}$ & 52.2 & -122.9 \\
\hline 5 & $1,4-\left(\mathrm{CH}_{3}\right) \mathrm{C}_{6} \mathrm{H}_{4}(\mathrm{OH}) \rightarrow 1,2-\left(\mathrm{CH}_{3}\right) \mathrm{C}_{6} \mathrm{H}_{4}(\mathrm{OH})$ & -5.0 & -123.6 \\
\hline & & average: & $-123.2 \pm 3.0$ \\
\hline \multicolumn{4}{|c|}{ 2,3-dimethylphenol } \\
\hline 1 & $2,3-\left(\mathrm{CH}_{3}\right)_{2} \mathrm{C}_{6} \mathrm{H}_{3}(\mathrm{OH})+2 \mathrm{CH}_{4} \rightarrow \mathrm{C}_{6} \mathrm{H}_{5} \mathrm{CH}_{3}+\mathrm{CH}_{3} \mathrm{OH}+\mathrm{CH}_{3} \mathrm{CH}_{3}$ & 73.1 & -158.6 \\
\hline 2 & $2,3-\left(\mathrm{CH}_{3}\right)_{2} \mathrm{C}_{6} \mathrm{H}_{3}(\mathrm{OH})+\mathrm{CH}_{3} \mathrm{CH}_{3} \rightarrow 1,2-\left(\mathrm{CH}_{3}\right) \mathrm{C}_{6} \mathrm{H}_{4}(\mathrm{OH})+\mathrm{CH}_{3} \mathrm{CH}_{2} \mathrm{CH}_{3}$ & 9.4 & -158.5 \\
\hline 3 & $2,3-\left(\mathrm{CH}_{3}\right)_{2} \mathrm{C}_{6} \mathrm{H}_{3}(\mathrm{OH})+\mathrm{CH}_{3} \mathrm{CH}_{3} \rightarrow \mathrm{C}_{6} \mathrm{H}_{5} \mathrm{OH}+\mathrm{CH}_{3} \mathrm{CH}_{2} \mathrm{CH}_{2} \mathrm{CH}_{3}$ & 24.8 & -158.5 \\
\hline 4 & $2,3-\left(\mathrm{CH}_{3}\right)_{2} \mathrm{C}_{6} \mathrm{H}_{3}(\mathrm{OH})+\mathrm{CH}_{3} \mathrm{CH}_{3} \rightarrow \mathrm{C}_{6} \mathrm{H}_{6}+\mathrm{CH}_{3} \mathrm{CH}_{2} \mathrm{OCH}_{2} \mathrm{CH}_{3}$ & 72.6 & -158.0 \\
\hline 5 & $2,3-\left(\mathrm{CH}_{3}\right)_{2} \mathrm{C}_{6} \mathrm{H}_{3}(\mathrm{OH})+\mathrm{CH}_{3} \mathrm{CH}_{3} \rightarrow \mathrm{C}_{6} \mathrm{H}_{5} \mathrm{OCH}_{3}+\mathrm{CH}_{3} \mathrm{CH}_{2} \mathrm{CH}_{3}$ & 66.6 & -157.8 \\
\hline & & average: & $-158.3 \pm 3.0$ \\
\hline \multicolumn{4}{|c|}{ 2,4-dimethylphenol } \\
\hline 1 & $2,4-\left(\mathrm{CH}_{3}\right)_{2} \mathrm{C}_{6} \mathrm{H}_{3}(\mathrm{OH})+2 \mathrm{CH}_{4} \rightarrow \mathrm{C}_{6} \mathrm{H}_{5} \mathrm{CH}_{3}+\mathrm{CH}_{3} \mathrm{OH}+\mathrm{CH}_{3} \mathrm{CH}_{3}$ & 74.3 & -159.8 \\
\hline 2 & $2,4-\left(\mathrm{CH}_{3}\right)_{2} \mathrm{C}_{6} \mathrm{H}_{3}(\mathrm{OH})+\mathrm{CH}_{3} \mathrm{CH}_{3} \rightarrow 1,2-\left(\mathrm{CH}_{3}\right) \mathrm{C}_{6} \mathrm{H}_{4}(\mathrm{OH})+\mathrm{CH}_{3} \mathrm{CH}_{2} \mathrm{CH}_{3}$ & 10.6 & -159.7 \\
\hline 3 & $2,4-\left(\mathrm{CH}_{3}\right)_{2} \mathrm{C}_{6} \mathrm{H}_{3}(\mathrm{OH})+\mathrm{CH}_{3} \mathrm{CH}_{3} \rightarrow \mathrm{C}_{6} \mathrm{H}_{5} \mathrm{OH}+\mathrm{CH}_{3} \mathrm{CH}_{2} \mathrm{CH}_{2} \mathrm{CH}_{3}$ & 26.0 & -159.7 \\
\hline 4 & $2,4-\left(\mathrm{CH}_{3}\right)_{2} \mathrm{C}_{6} \mathrm{H}_{3}(\mathrm{OH})+\mathrm{CH}_{3} \mathrm{CH}_{3} \rightarrow \mathrm{C}_{6} \mathrm{H}_{6}+\mathrm{CH}_{3} \mathrm{CH}_{2} \mathrm{OCH}_{2} \mathrm{CH}_{3}$ & 73.8 & -159.2 \\
\hline 5 & $2,4-\left(\mathrm{CH}_{3}\right)_{2} \mathrm{C}_{6} \mathrm{H}_{3}(\mathrm{OH})+\mathrm{CH}_{3} \mathrm{CH}_{3} \rightarrow \mathrm{C}_{6} \mathrm{H}_{5} \mathrm{OCH}_{3}+\mathrm{CH}_{3} \mathrm{CH}_{2} \mathrm{CH}_{3}$ & 67.8 & -159.0 \\
\hline 6 & $2,4-\left(\mathrm{CH}_{3}\right)_{2} \mathrm{C}_{6} \mathrm{H}_{3}(\mathrm{OH}) \rightarrow 2,3-\left(\mathrm{CH}_{3}\right)_{2} \mathrm{C}_{6} \mathrm{H}_{3}(\mathrm{OH})$ & 1.2 & -158.4 \\
\hline & & average: & $-159.3 \pm 3.0$ \\
\hline \multicolumn{4}{|c|}{ 2,5-dimethylphenol } \\
\hline 1 & $2,5-\left(\mathrm{CH}_{3}\right)_{2} \mathrm{C}_{6} \mathrm{H}_{3}(\mathrm{OH})+2 \mathrm{CH}_{4} \rightarrow \mathrm{C}_{6} \mathrm{H}_{5} \mathrm{CH}_{3}+\mathrm{CH}_{3} \mathrm{OH}+\mathrm{CH}_{3} \mathrm{CH}_{3}$ & 76.0 & -161.5 \\
\hline 2 & $2,5-\left(\mathrm{CH}_{3}\right)_{2} \mathrm{C}_{6} \mathrm{H}_{3}(\mathrm{OH})+\mathrm{CH}_{3} \mathrm{CH}_{3} \rightarrow 1,2-\left(\mathrm{CH}_{3}\right) \mathrm{C}_{6} \mathrm{H}_{4}(\mathrm{OH})+\mathrm{CH}_{3} \mathrm{CH}_{2} \mathrm{CH}_{3}$ & 12.3 & -161.4 \\
\hline 3 & $2,5-\left(\mathrm{CH}_{3}\right)_{2} \mathrm{C}_{6} \mathrm{H}_{3}(\mathrm{OH})+\mathrm{CH}_{3} \mathrm{CH}_{3} \rightarrow \mathrm{C}_{6} \mathrm{H}_{5} \mathrm{OH}+\mathrm{CH}_{3} \mathrm{CH}_{2} \mathrm{CH}_{2} \mathrm{CH}_{3}$ & 27.7 & -161.4 \\
\hline 4 & $2,5-\left(\mathrm{CH}_{3}\right)_{2} \mathrm{C}_{6} \mathrm{H}_{3}(\mathrm{OH})+\mathrm{CH}_{3} \mathrm{CH}_{3} \rightarrow \mathrm{C}_{6} \mathrm{H}_{6}+\mathrm{CH}_{3} \mathrm{CH}_{2} \mathrm{OCH}_{2} \mathrm{CH}_{3}$ & 75.5 & -160.9 \\
\hline 5 & $2,5-\left(\mathrm{CH}_{3}\right)_{2} \mathrm{C}_{6} \mathrm{H}_{3}(\mathrm{OH})+\mathrm{CH}_{3} \mathrm{CH}_{3} \rightarrow \mathrm{C}_{6} \mathrm{H}_{5} \mathrm{OCH}_{3}+\mathrm{CH}_{3} \mathrm{CH}_{2} \mathrm{CH}_{3}$ & 69.5 & -160.7 \\
\hline 6 & $2,5-\left(\mathrm{CH}_{3}\right)_{2} \mathrm{C}_{6} \mathrm{H}_{3}(\mathrm{OH}) \rightarrow 2,3-\left(\mathrm{CH}_{3}\right)_{2} \mathrm{C}_{6} \mathrm{H}_{3}(\mathrm{OH})$ & 2.9 & -160.1 \\
\hline & & average: & $-161.0 \pm 3.0$ \\
\hline \multicolumn{4}{|c|}{ 2,6-dimethylphenol } \\
\hline 1 & $2,6-\left(\mathrm{CH}_{3}\right)_{2} \mathrm{C}_{6} \mathrm{H}_{3}(\mathrm{OH})+2 \mathrm{CH}_{4} \rightarrow \mathrm{C}_{6} \mathrm{H}_{5} \mathrm{CH}_{3}+\mathrm{CH}_{3} \mathrm{OH}+\mathrm{CH}_{3} \mathrm{CH}_{3}$ & 77.7 & -163.2 \\
\hline 2 & $2,6-\left(\mathrm{CH}_{3}\right)_{2} \mathrm{C}_{6} \mathrm{H}_{3}(\mathrm{OH})+\mathrm{CH}_{3} \mathrm{CH}_{3} \rightarrow 1,2-\left(\mathrm{CH}_{3}\right) \mathrm{C}_{6} \mathrm{H}_{4}(\mathrm{OH})+\mathrm{CH}_{3} \mathrm{CH}_{2} \mathrm{CH}_{3}$ & 14.0 & -163.1 \\
\hline 3 & $2,6-\left(\mathrm{CH}_{3}\right)_{2} \mathrm{C}_{6} \mathrm{H}_{3}(\mathrm{OH})+\mathrm{CH}_{3} \mathrm{CH}_{3} \rightarrow \mathrm{C}_{6} \mathrm{H}_{5} \mathrm{OH}+\mathrm{CH}_{3} \mathrm{CH}_{2} \mathrm{CH}_{2} \mathrm{CH}_{3}$ & 29.3 & -163.1 \\
\hline 4 & $2,6-\left(\mathrm{CH}_{3}\right)_{2} \mathrm{C}_{6} \mathrm{H}_{3}(\mathrm{OH})+\mathrm{CH}_{3} \mathrm{CH}_{3} \rightarrow \mathrm{C}_{6} \mathrm{H}_{6}+\mathrm{CH}_{3} \mathrm{CH}_{2} \mathrm{OCH}_{2} \mathrm{CH}_{3}$ & 77.1 & -162.5 \\
\hline
\end{tabular}




\begin{tabular}{|c|c|c|c|}
\hline 5 & 2,6- $\left(\mathrm{CH}_{3}\right)_{2} \mathrm{C}_{6} \mathrm{H}_{3}(\mathrm{OH})+\mathrm{CH}_{3} \mathrm{CH}_{3} \rightarrow \mathrm{C}_{6} \mathrm{H}_{5} \mathrm{OCH}_{3}+\mathrm{CH}_{3} \mathrm{CH}_{2} \mathrm{CH}_{3}$ & 71.1 & -162.3 \\
\hline 6 & $2,6-\left(\mathrm{CH}_{3}\right)_{2} \mathrm{C}_{6} \mathrm{H}_{3}(\mathrm{OH}) \rightarrow 2,3-\left(\mathrm{CH}_{3}\right)_{2} \mathrm{C}_{6} \mathrm{H}_{3}(\mathrm{OH})$ & 4.6 & -161.8 \\
\hline 7 & $2,6-\left(\mathrm{CH}_{3}\right)_{2} \mathrm{C}_{6} \mathrm{H}_{3}(\mathrm{OH}) \rightarrow 2,5-\left(\mathrm{CH}_{3}\right)_{2} \mathrm{C}_{6} \mathrm{H}_{3}(\mathrm{OH})$ & 1.7 & -163.3 \\
\hline & & average: & $-162.7 \pm 3.0$ \\
\hline \multicolumn{4}{|c|}{ 3,4-dimethylphenol } \\
\hline 1 & $3,4-\left(\mathrm{CH}_{3}\right)_{2} \mathrm{C}_{6} \mathrm{H}_{3}(\mathrm{OH})+2 \mathrm{CH}_{4} \rightarrow \mathrm{C}_{6} \mathrm{H}_{5} \mathrm{CH}_{3}+\mathrm{CH}_{3} \mathrm{OH}+\mathrm{CH}_{3} \mathrm{CH}_{3}$ & 72.8 & -158.3 \\
\hline 2 & 3,4- $\left(\mathrm{CH}_{3}\right)_{2} \mathrm{C}_{6} \mathrm{H}_{3}(\mathrm{OH})+\mathrm{CH}_{3} \mathrm{CH}_{3} \rightarrow 1,2-\left(\mathrm{CH}_{3}\right) \mathrm{C}_{6} \mathrm{H}_{4}(\mathrm{OH})+\mathrm{CH}_{3} \mathrm{CH}_{2} \mathrm{CH}_{3}$ & 9.1 & -158.2 \\
\hline 3 & $3,4-\left(\mathrm{CH}_{3}\right)_{2} \mathrm{C}_{6} \mathrm{H}_{3}(\mathrm{OH})+\mathrm{CH}_{3} \mathrm{CH}_{3} \rightarrow \mathrm{C}_{6} \mathrm{H}_{5} \mathrm{OH}+\mathrm{CH}_{3} \mathrm{CH}_{2} \mathrm{CH}_{2} \mathrm{CH}_{3}$ & 24.4 & -158.2 \\
\hline 4 & $3,4-\left(\mathrm{CH}_{3}\right)_{2} \mathrm{C}_{6} \mathrm{H}_{3}(\mathrm{OH})+\mathrm{CH}_{3} \mathrm{CH}_{3} \rightarrow \mathrm{C}_{6} \mathrm{H}_{6}+\mathrm{CH}_{3} \mathrm{CH}_{2} \mathrm{OCH}_{2} \mathrm{CH}_{3}$ & 72.3 & -157.7 \\
\hline 5 & $3,4-\left(\mathrm{CH}_{3}\right)_{2} \mathrm{C}_{6} \mathrm{H}_{3}(\mathrm{OH})+\mathrm{CH}_{3} \mathrm{CH}_{3} \rightarrow \mathrm{C}_{6} \mathrm{H}_{5} \mathrm{OCH}_{3}+\mathrm{CH}_{3} \mathrm{CH}_{2} \mathrm{CH}_{3}$ & 66.2 & -157.4 \\
\hline 6 & $3,4-\left(\mathrm{CH}_{3}\right)_{2} \mathrm{C}_{6} \mathrm{H}_{3}(\mathrm{OH}) \rightarrow 2,3-\left(\mathrm{CH}_{3}\right)_{2} \mathrm{C}_{6} \mathrm{H}_{3}(\mathrm{OH})$ & -0.3 & -156.9 \\
\hline 7 & $3,4-\left(\mathrm{CH}_{3}\right)_{2} \mathrm{C}_{6} \mathrm{H}_{3}(\mathrm{OH}) \rightarrow 2,5-\left(\mathrm{CH}_{3}\right)_{2} \mathrm{C}_{6} \mathrm{H}_{3}(\mathrm{OH})$ & -3.2 & -158.4 \\
\hline 8 & $3,4-\left(\mathrm{CH}_{3}\right)_{2} \mathrm{C}_{6} \mathrm{H}_{3}(\mathrm{OH}) \rightarrow 2,6-\left(\mathrm{CH}_{3}\right)_{2} \mathrm{C}_{6} \mathrm{H}_{3}(\mathrm{OH})$ & -4.9 & -157.2 \\
\hline & & average: & $-157.8 \pm 3.0$ \\
\hline \multicolumn{4}{|c|}{ 3,5-dimethylphenol } \\
\hline 1 & $3,5-\left(\mathrm{CH}_{3}\right)_{2} \mathrm{C}_{6} \mathrm{H}_{3}(\mathrm{OH})+2 \mathrm{CH}_{4} \rightarrow \mathrm{C}_{6} \mathrm{H}_{5} \mathrm{CH}_{3}+\mathrm{CH}_{3} \mathrm{OH}+\mathrm{CH}_{3} \mathrm{CH}_{3}$ & 74.2 & -159.7 \\
\hline 2 & $3,5-\left(\mathrm{CH}_{3}\right)_{2} \mathrm{C}_{6} \mathrm{H}_{3}(\mathrm{OH})+\mathrm{CH}_{3} \mathrm{CH}_{3} \rightarrow 1,2-\left(\mathrm{CH}_{3}\right) \mathrm{C}_{6} \mathrm{H}_{4}(\mathrm{OH})+\mathrm{CH}_{3} \mathrm{CH}_{2} \mathrm{CH}_{3}$ & 10.5 & -159.6 \\
\hline 3 & $3,5-\left(\mathrm{CH}_{3}\right)_{2} \mathrm{C}_{6} \mathrm{H}_{3}(\mathrm{OH})+\mathrm{CH}_{3} \mathrm{CH}_{3} \rightarrow \mathrm{C}_{6} \mathrm{H}_{5} \mathrm{OH}+\mathrm{CH}_{3} \mathrm{CH}_{2} \mathrm{CH}_{2} \mathrm{CH}_{3}$ & 25.8 & -159.6 \\
\hline 4 & 3,5-( $\left(\mathrm{CH}_{3}\right)_{2} \mathrm{C}_{6} \mathrm{H}_{3}(\mathrm{OH})+\mathrm{CH}_{3} \mathrm{CH}_{3} \rightarrow \mathrm{C}_{6} \mathrm{H}_{6}+\mathrm{CH}_{3} \mathrm{CH}_{2} \mathrm{OCH}_{2} \mathrm{CH}_{3}$ & 73.7 & -159.1 \\
\hline 5 & $3,5-\left(\mathrm{CH}_{3}\right)_{2} \mathrm{C}_{6} \mathrm{H}_{3}(\mathrm{OH})+\mathrm{CH}_{3} \mathrm{CH}_{3} \rightarrow \mathrm{C}_{6} \mathrm{H}_{5} \mathrm{OCH}_{3}+\mathrm{CH}_{3} \mathrm{CH}_{2} \mathrm{CH}_{3}$ & 67.6 & -158.8 \\
\hline 6 & $3,5-\left(\mathrm{CH}_{3}\right)_{2} \mathrm{C}_{6} \mathrm{H}_{3}(\mathrm{OH}) \rightarrow 2,3-\left(\mathrm{CH}_{3}\right)_{2} \mathrm{C}_{6} \mathrm{H}_{3}(\mathrm{OH})$ & 1.1 & -158.3 \\
\hline 7 & $3,5-\left(\mathrm{CH}_{3}\right)_{2} \mathrm{C}_{6} \mathrm{H}_{3}(\mathrm{OH}) \rightarrow 2,5-\left(\mathrm{CH}_{3}\right)_{2} \mathrm{C}_{6} \mathrm{H}_{3}(\mathrm{OH})$ & -1.8 & -159.8 \\
\hline 8 & $3,5-\left(\mathrm{CH}_{3}\right)_{2} \mathrm{C}_{6} \mathrm{H}_{3}(\mathrm{OH}) \rightarrow 2,6-\left(\mathrm{CH}_{3}\right)_{2} \mathrm{C}_{6} \mathrm{H}_{3}(\mathrm{OH})$ & -3.5 & -158.6 \\
\hline 9 & $3,5-\left(\mathrm{CH}_{3}\right)_{2} \mathrm{C}_{6} \mathrm{H}_{3}(\mathrm{OH}) \rightarrow 3,4-\left(\mathrm{CH}_{3}\right)_{2} \mathrm{C}_{6} \mathrm{H}_{3}(\mathrm{OH})$ & 1.4 & -158.7 \\
\hline & & average: & $-159.1 \pm 3.0$ \\
\hline \multicolumn{4}{|c|}{ 2,3,6-trimethylphenol } \\
\hline 1 & $2,3,6-\left(\mathrm{CH}_{3}\right)_{3} \mathrm{C}_{6} \mathrm{H}_{2}(\mathrm{OH})+2 \mathrm{CH}_{4} \rightarrow \mathrm{C}_{6} \mathrm{H}_{5} \mathrm{CH}_{3}+\mathrm{CH}_{3} \mathrm{CH}_{2} \mathrm{OH}+\mathrm{CH}_{3} \mathrm{CH}_{3}$ & 74.8 & -194.2 \\
\hline 2 & $2,3,6-\left(\mathrm{CH}_{3}\right)_{3} \mathrm{C}_{6} \mathrm{H}_{2}(\mathrm{OH})+\mathrm{CH}_{4} \rightarrow 1,2-\left(\mathrm{CH}_{3}\right) \mathrm{C}_{6} \mathrm{H}_{4}(\mathrm{OH})+\mathrm{CH}_{3} \mathrm{CH}_{2} \mathrm{CH}_{3}$ & 34.6 & -193.1 \\
\hline 3 & $2,3,6-\left(\mathrm{CH}_{3}\right)_{3} \mathrm{C}_{6} \mathrm{H}_{2}(\mathrm{OH})+\mathrm{CH}_{4} \rightarrow \mathrm{C}_{6} \mathrm{H}_{5} \mathrm{OH}+\mathrm{CH}_{3} \mathrm{CH}_{2} \mathrm{CH}_{2} \mathrm{CH}_{3}$ & 49.9 & -193.1 \\
\hline 4 & $2,3,6-\left(\mathrm{CH}_{3}\right)_{3} \mathrm{C}_{6} \mathrm{H}_{2}(\mathrm{OH})+\mathrm{CH}_{4} \rightarrow \mathrm{C}_{6} \mathrm{H}_{6}+\mathrm{CH}_{3} \mathrm{CH}_{2} \mathrm{OCH}_{2} \mathrm{CH}_{3}$ & 97.8 & -192.6 \\
\hline 5 & $2,3,6-\left(\mathrm{CH}_{3}\right)_{3} \mathrm{C}_{6} \mathrm{H}_{2}(\mathrm{OH})+\mathrm{CH}_{4} \rightarrow \mathrm{C}_{6} \mathrm{H}_{5} \mathrm{OCH}_{3}+\mathrm{CH}_{3} \mathrm{CH}_{2} \mathrm{CH}_{3}$ & 91.7 & -192.3 \\
\hline 6 & $2,3,6-\left(\mathrm{CH}_{3}\right)_{3} \mathrm{C}_{6} \mathrm{H}_{2}(\mathrm{OH})+\mathrm{CH}_{3} \mathrm{CH}_{3} \rightarrow 2,3-\left(\mathrm{CH}_{3}\right)_{2} \mathrm{C}_{6} \mathrm{H}_{3}(\mathrm{OH})+\mathrm{CH}_{3} \mathrm{CH}_{2} \mathrm{CH}_{3}$ & 13.6 & -191.3 \\
\hline 7 & $2,3,6-\left(\mathrm{CH}_{3}\right)_{3} \mathrm{C}_{6} \mathrm{H}_{2}(\mathrm{OH})+\mathrm{CH}_{3} \mathrm{CH}_{3} \rightarrow 2,5-\left(\mathrm{CH}_{3}\right)_{2} \mathrm{C}_{6} \mathrm{H}_{3}(\mathrm{OH})+\mathrm{CH}_{3} \mathrm{CH}_{2} \mathrm{CH}_{3}$ & 10.7 & -192.8 \\
\hline 8 & $2,3,6-\left(\mathrm{CH}_{3}\right)_{3} \mathrm{C}_{6} \mathrm{H}_{2}(\mathrm{OH})+\mathrm{CH}_{3} \mathrm{CH}_{3} \rightarrow 2,6-\left(\mathrm{CH}_{3}\right)_{2} \mathrm{C}_{6} \mathrm{H}_{3}(\mathrm{OH})+\mathrm{CH}_{3} \mathrm{CH}_{2} \mathrm{CH}_{3}$ & 9.0 & -191.6 \\
\hline 9 & $2,3,6-\left(\mathrm{CH}_{3}\right)_{3} \mathrm{C}_{6} \mathrm{H}_{2}(\mathrm{OH})+\mathrm{CH}_{3} \mathrm{CH}_{3} \rightarrow 3,4-\left(\mathrm{CH}_{3}\right)_{2} \mathrm{C}_{6} \mathrm{H}_{3}(\mathrm{OH})+\mathrm{CH}_{3} \mathrm{CH}_{2} \mathrm{CH}_{3}$ & 13.9 & -191.7 \\
\hline & & average: & $-192.5 \pm 3.0$ \\
\hline \multicolumn{4}{|c|}{ 2,4,6-trimethylphenol } \\
\hline 1 & $2,4,6-\left(\mathrm{CH}_{3}\right)_{3} \mathrm{C}_{6} \mathrm{H}_{2}(\mathrm{OH})+2 \mathrm{CH}_{4} \rightarrow \mathrm{C}_{6} \mathrm{H}_{5} \mathrm{CH}_{3}+\mathrm{CH}_{3} \mathrm{CH}_{2} \mathrm{OH}+\mathrm{CH}_{3} \mathrm{CH}_{3}$ & 76.5 & -195.9 \\
\hline 2 & $2,4,6-\left(\mathrm{CH}_{3}\right)_{3} \mathrm{C}_{6} \mathrm{H}_{2}(\mathrm{OH})+\mathrm{CH}_{4} \rightarrow 1,2-\left(\mathrm{CH}_{3}\right) \mathrm{C}_{6} \mathrm{H}_{4}(\mathrm{OH})+\mathrm{CH}_{3} \mathrm{CH}_{2} \mathrm{CH}_{3}$ & 36.3 & -194.8 \\
\hline 3 & $2,4,6-\left(\mathrm{CH}_{3}\right)_{3} \mathrm{C}_{6} \mathrm{H}_{2}(\mathrm{OH})+\mathrm{CH}_{4} \rightarrow \mathrm{C}_{6} \mathrm{H}_{5} \mathrm{OH}+\mathrm{CH}_{3} \mathrm{CH}_{2} \mathrm{CH}_{2} \mathrm{CH}_{3}$ & 51.7 & -194.8 \\
\hline 4 & $2,4,6-\left(\mathrm{CH}_{3}\right)_{3} \mathrm{C}_{6} \mathrm{H}_{2}(\mathrm{OH})+\mathrm{CH}_{4} \rightarrow \mathrm{C}_{6} \mathrm{H}_{6}+\mathrm{CH}_{3} \mathrm{CH}_{2} \mathrm{OCH}_{2} \mathrm{CH}_{3}$ & 99.5 & -194.3 \\
\hline 5 & $2,4,6-\left(\mathrm{CH}_{3}\right)_{3} \mathrm{C}_{6} \mathrm{H}_{2}(\mathrm{OH})+\mathrm{CH}_{4} \rightarrow \mathrm{C}_{6} \mathrm{H}_{5} \mathrm{OCH}_{3}+\mathrm{CH}_{3} \mathrm{CH}_{2} \mathrm{CH}_{3}$ & 93.4 & -194.0 \\
\hline 6 & $2,4,6-\left(\mathrm{CH}_{3}\right)_{3} \mathrm{C}_{6} \mathrm{H}_{2}(\mathrm{OH})+\mathrm{CH}_{3} \mathrm{CH}_{3} \rightarrow 2,3-\left(\mathrm{CH}_{3}\right)_{2} \mathrm{C}_{6} \mathrm{H}_{3}(\mathrm{OH})+\mathrm{CH}_{3} \mathrm{CH}_{2} \mathrm{CH}_{3}$ & 15.3 & -193.0 \\
\hline 7 & $2,4,6-\left(\mathrm{CH}_{3}\right)_{3} \mathrm{C}_{6} \mathrm{H}_{2}(\mathrm{OH})+\mathrm{CH}_{3} \mathrm{CH}_{3} \rightarrow 2,5-\left(\mathrm{CH}_{3}\right)_{2} \mathrm{C}_{6} \mathrm{H}_{3}(\mathrm{OH})+\mathrm{CH}_{3} \mathrm{CH}_{2} \mathrm{CH}_{3}$ & 12.4 & -194.5 \\
\hline 8 & $2,4,6-\left(\mathrm{CH}_{3}\right)_{3} \mathrm{C}_{6} \mathrm{H}_{2}(\mathrm{OH})+\mathrm{CH}_{3} \mathrm{CH}_{3} \rightarrow 2,6-\left(\mathrm{CH}_{3}\right)_{2} \mathrm{C}_{6} \mathrm{H}_{3}(\mathrm{OH})+\mathrm{CH}_{3} \mathrm{CH}_{2} \mathrm{CH}_{3}$ & 10.7 & -193.3 \\
\hline 9 & $2,4,6-\left(\mathrm{CH}_{3}\right)_{3} \mathrm{C}_{6} \mathrm{H}_{2}(\mathrm{OH})+\mathrm{CH}_{3} \mathrm{CH}_{3} \rightarrow 3,4-\left(\mathrm{CH}_{3}\right)_{2} \mathrm{C}_{6} \mathrm{H}_{3}(\mathrm{OH})+\mathrm{CH}_{3} \mathrm{CH}_{2} \mathrm{CH}_{3}$ & 15.6 & -193.4 \\
\hline 10 & $2,4,6-\left(\mathrm{CH}_{3}\right)_{3} \mathrm{C}_{6} \mathrm{H}_{2}(\mathrm{OH}) \rightarrow 2,3,6-\left(\mathrm{CH}_{3}\right)_{3} \mathrm{C}_{6} \mathrm{H}_{2}(\mathrm{OH})$ & 1.7 & -194.6 \\
\hline & & average: & $-194.3 \pm 3.0$ \\
\hline \multicolumn{4}{|c|}{ 1,2-dihydroxybenzene } \\
\hline 1 & $1,2-\mathrm{C}_{6} \mathrm{H}_{4}(\mathrm{OH})_{2}+\mathrm{C}_{6} \mathrm{H}_{6} \rightarrow 2 \mathrm{C}_{6} \mathrm{H}_{5} \mathrm{OH}$ & 2.2 & -268.7 \\
\hline 2 & $1,2-\mathrm{C}_{6} \mathrm{H}_{4}(\mathrm{OH})_{2}+2 \mathrm{CH}_{4} \rightarrow 2 \mathrm{CH}_{3} \mathrm{OH}+\mathrm{C}_{6} \mathrm{H}_{6}$ & 100.5 & -270.0 \\
\hline 3 & $1,2-\mathrm{C}_{6} \mathrm{H}_{4}(\mathrm{OH})_{2}+\mathrm{CH}_{3} \mathrm{CH}_{3} \rightarrow 1,2-\left(\mathrm{CH}_{3}\right) \mathrm{C}_{6} \mathrm{H}_{4}(\mathrm{OH})+\mathrm{CH}_{3} \mathrm{OH}$ & 23.7 & -269.1 \\
\hline 4 & $1,2-\mathrm{C}_{6} \mathrm{H}_{4}(\mathrm{OH})_{2}+\mathrm{CH}_{3} \mathrm{CH}_{3} \rightarrow \mathrm{C}_{6} \mathrm{H}_{5} \mathrm{OCH}_{3}+\mathrm{CH}_{3} \mathrm{OH}$ & 80.8 & -268.3 \\
\hline 5 & $1,2-\mathrm{C}_{6} \mathrm{H}_{4}(\mathrm{OH})_{2}+\mathrm{CH}_{3} \mathrm{CH}_{2} \mathrm{CH}_{3} \rightarrow 2,3-\left(\mathrm{CH}_{3}\right)_{2} \mathrm{C}_{6} \mathrm{H}_{3}(\mathrm{OH})+\mathrm{CH}_{3} \mathrm{OH}$ & 14.3 & -267.8 \\
\hline 6 & $1,2-\mathrm{C}_{6} \mathrm{H}_{4}(\mathrm{OH})_{2}+\mathrm{CH}_{3} \mathrm{CH}_{2} \mathrm{CH}_{3} \rightarrow 2,5-\left(\mathrm{CH}_{3}\right)_{2} \mathrm{C}_{6} \mathrm{H}_{3}(\mathrm{OH})+\mathrm{CH}_{3} \mathrm{OH}$ & 11.4 & -269.3 \\
\hline
\end{tabular}




\begin{tabular}{|c|c|c|c|}
\hline 7 & $1,2-\mathrm{C}_{6} \mathrm{H}_{4}(\mathrm{OH})_{2}+\mathrm{CH}_{3} \mathrm{CH}_{2} \mathrm{CH}_{3} \rightarrow 2,6-\left(\mathrm{CH}_{3}\right)_{2} \mathrm{C}_{6} \mathrm{H}_{3}(\mathrm{OH})+\mathrm{CH}_{3} \mathrm{OH}$ & 9.7 & -268.1 \\
\hline 8 & $1,2-\mathrm{C}_{6} \mathrm{H}_{4}(\mathrm{OH})_{2}+\mathrm{CH}_{3} \mathrm{CH}_{2} \mathrm{CH}_{3} \rightarrow 3,4-\left(\mathrm{CH}_{3}\right)_{2} \mathrm{C}_{6} \mathrm{H}_{3}(\mathrm{OH})+\mathrm{CH}_{3} \mathrm{OH}$ & 14.6 & -268.2 \\
\hline 9 & $1,2-\mathrm{C}_{6} \mathrm{H}_{4}(\mathrm{OH})_{2}+\mathrm{CH}_{3} \mathrm{CH}_{2} \mathrm{CH}_{2} \mathrm{CH}_{3} \rightarrow 2,3,6-\left(\mathrm{CH}_{3}\right)_{3} \mathrm{C}_{6} \mathrm{H}_{2}(\mathrm{OH})+\mathrm{CH}_{3} \mathrm{OH}$ & 1.4 & -269.1 \\
\hline 10 & $1,2-\mathrm{C}_{6} \mathrm{H}_{4}(\mathrm{OH})_{2}+\mathrm{CH}_{3} \mathrm{CH}_{3} \rightarrow \mathrm{C}_{6} \mathrm{H}_{6}+\mathrm{HOCH}_{2} \mathrm{CH}_{2} \mathrm{OH}$ & 47.9 & -270.5 \\
\hline 11 & $1,2-\mathrm{C}_{6} \mathrm{H}_{4}(\mathrm{OH})_{2}+\mathrm{CH}_{4} \rightarrow \mathrm{C}_{6} \mathrm{H}_{6}+\mathrm{CH}_{2}(\mathrm{OH})_{2}$ & 35.6 & -270.8 \\
\hline 12 & $1,2-\mathrm{C}_{6} \mathrm{H}_{4}(\mathrm{OH})_{2}+\mathrm{CH}_{3} \mathrm{CH}_{2} \mathrm{CH}_{3} \rightarrow \mathrm{C}_{6} \mathrm{H}_{6}+\mathrm{HOCH}_{2} \mathrm{CH}_{2} \mathrm{CH}_{2} \mathrm{OH}$ & 47.7 & -271.0 \\
\hline 13 & $1,2-\mathrm{C}_{6} \mathrm{H}_{4}(\mathrm{OH})_{2}+\mathrm{CH}_{3} \mathrm{CH}_{2} \mathrm{CH}_{3} \rightarrow \mathrm{C}_{6} \mathrm{H}_{6}+\mathrm{HOCH}_{2} \mathrm{CH}(\mathrm{OH}) \mathrm{CH}_{3}$ & $\begin{array}{l}28.3 \\
\text { average: }\end{array}$ & $\begin{array}{l}-269.4 \\
\mathbf{- 2 6 9 . 2} \pm \mathbf{3 . 0}\end{array}$ \\
\hline \multicolumn{4}{|c|}{ 1,3-dihydroxybenzene } \\
\hline 1 & $1,3-\mathrm{C}_{6} \mathrm{H}_{4}(\mathrm{OH})_{2}+\mathrm{C}_{6} \mathrm{H}_{6} \rightarrow 2 \mathrm{C}_{6} \mathrm{H}_{5} \mathrm{OH}$ & 0.9 & -267.4 \\
\hline 2 & $1,3-\mathrm{C}_{6} \mathrm{H}_{4}(\mathrm{OH})_{2}+2 \mathrm{CH}_{4} \rightarrow 2 \mathrm{CH}_{3} \mathrm{OH}+\mathrm{C}_{6} \mathrm{H}_{6}$ & 99.2 & -268.7 \\
\hline 3 & $1,3-\mathrm{C}_{6} \mathrm{H}_{4}(\mathrm{OH})_{2}+\mathrm{CH}_{3} \mathrm{CH}_{3} \rightarrow 1,2-\left(\mathrm{CH}_{3}\right) \mathrm{C}_{6} \mathrm{H}_{4}(\mathrm{OH})+\mathrm{CH}_{3} \mathrm{OH}$ & 22.4 & -267.8 \\
\hline 4 & $1,3-\mathrm{C}_{6} \mathrm{H}_{4}(\mathrm{OH})_{2}+\mathrm{CH}_{3} \mathrm{CH}_{3} \rightarrow \mathrm{C}_{6} \mathrm{H}_{5} \mathrm{OCH}_{3}+\mathrm{CH}_{3} \mathrm{OH}$ & 79.6 & -267.1 \\
\hline 5 & $1,3-\mathrm{C}_{6} \mathrm{H}_{4}(\mathrm{OH})_{2}+\mathrm{CH}_{3} \mathrm{CH}_{2} \mathrm{CH}_{3} \rightarrow 2,3-\left(\mathrm{CH}_{3}\right)_{2} \mathrm{C}_{6} \mathrm{H}_{3}(\mathrm{OH})+\mathrm{CH}_{3} \mathrm{OH}$ & 13.0 & -266.5 \\
\hline 6 & $1,3-\mathrm{C}_{6} \mathrm{H}_{4}(\mathrm{OH})_{2}+\mathrm{CH}_{3} \mathrm{CH}_{2} \mathrm{CH}_{3} \rightarrow 2,5-\left(\mathrm{CH}_{3}\right)_{2} \mathrm{C}_{6} \mathrm{H}_{3}(\mathrm{OH})+\mathrm{CH}_{3} \mathrm{OH}$ & 10.1 & -268.0 \\
\hline 7 & $1,3-\mathrm{C}_{6} \mathrm{H}_{4}(\mathrm{OH})_{2}+\mathrm{CH}_{3} \mathrm{CH}_{2} \mathrm{CH}_{3} \rightarrow 2,6-\left(\mathrm{CH}_{3}\right)_{2} \mathrm{C}_{6} \mathrm{H}_{3}(\mathrm{OH})+\mathrm{CH}_{3} \mathrm{OH}$ & 8.5 & -266.9 \\
\hline 8 & $1,3-\mathrm{C}_{6} \mathrm{H}_{4}(\mathrm{OH})_{2}+\mathrm{CH}_{3} \mathrm{CH}_{2} \mathrm{CH}_{3} \rightarrow 3,4-\left(\mathrm{CH}_{3}\right)_{2} \mathrm{C}_{6} \mathrm{H}_{3}(\mathrm{OH})+\mathrm{CH}_{3} \mathrm{OH}$ & 13.3 & -266.9 \\
\hline 9 & $1,3-\mathrm{C}_{6} \mathrm{H}_{4}(\mathrm{OH})_{2}+\mathrm{CH}_{3} \mathrm{CH}_{2} \mathrm{CH}_{2} \mathrm{CH}_{3} \rightarrow 2,3,6-\left(\mathrm{CH}_{3}\right)_{3} \mathrm{C}_{6} \mathrm{H}_{2}(\mathrm{OH})+\mathrm{CH}_{3} \mathrm{OH}$ & 0.1 & -267.8 \\
\hline 10 & $1,3-\mathrm{C}_{6} \mathrm{H}_{4}(\mathrm{OH})_{2}+\mathrm{CH}_{3} \mathrm{CH}_{3} \rightarrow \mathrm{C}_{6} \mathrm{H}_{6}+\mathrm{HOCH}_{2} \mathrm{CH}_{2} \mathrm{OH}$ & 46.6 & -269.2 \\
\hline 11 & $1,3-\mathrm{C}_{6} \mathrm{H}_{4}(\mathrm{OH})_{2}+\mathrm{CH}_{4} \rightarrow \mathrm{C}_{6} \mathrm{H}_{6}+\mathrm{CH}_{2}(\mathrm{OH})_{2}$ & 34.3 & -269.5 \\
\hline 12 & $1,3-\mathrm{C}_{6} \mathrm{H}_{4}(\mathrm{OH})_{2}+\mathrm{CH}_{3} \mathrm{CH}_{2} \mathrm{CH}_{3} \rightarrow \mathrm{C}_{6} \mathrm{H}_{6}+\mathrm{HOCH}_{2} \mathrm{CH}_{2} \mathrm{CH}_{2} \mathrm{OH}$ & 46.4 & -269.7 \\
\hline 13 & $1,3-\mathrm{C}_{6} \mathrm{H}_{4}(\mathrm{OH})_{2}+\mathrm{CH}_{3} \mathrm{CH}_{2} \mathrm{CH}_{3} \rightarrow \mathrm{C}_{6} \mathrm{H}_{6}+\mathrm{HOCH}_{2} \mathrm{CH}(\mathrm{OH}) \mathrm{CH}_{3}$ & 27.0 & -268.1 \\
\hline 14 & $1,3-\mathrm{C}_{6} \mathrm{H}_{4}(\mathrm{OH})_{2} \rightarrow 1,2-\mathrm{C}_{6} \mathrm{H}_{4}(\mathrm{OH})_{2}$ & -1.3 & -267.8 \\
\hline \multicolumn{4}{|c|}{ 1,4-dihydroxybenzene } \\
\hline 1 & $1,4-\mathrm{C}_{6} \mathrm{H}_{4}(\mathrm{OH})_{2}+\mathrm{C}_{6} \mathrm{H}_{6} \rightarrow 2 \mathrm{C}_{6} \mathrm{H}_{5} \mathrm{OH}$ & -7.8 & -258.7 \\
\hline 2 & $1,4-\mathrm{C}_{6} \mathrm{H}_{4}(\mathrm{OH})_{2}+2 \mathrm{CH}_{4} \rightarrow 2 \mathrm{CH}_{3} \mathrm{OH}+\mathrm{C}_{6} \mathrm{H}_{6}$ & 90.5 & -260.0 \\
\hline 3 & $1,4-\mathrm{C}_{6} \mathrm{H}_{4}(\mathrm{OH})_{2}+\mathrm{CH}_{3} \mathrm{CH}_{3} \rightarrow 1,2-\left(\mathrm{CH}_{3}\right) \mathrm{C}_{6} \mathrm{H}_{4}(\mathrm{OH})+\mathrm{CH}_{3} \mathrm{OH}$ & 13.7 & -259.1 \\
\hline 4 & $1,4-\mathrm{C}_{6} \mathrm{H}_{4}(\mathrm{OH})_{2}+\mathrm{CH}_{3} \mathrm{CH}_{3} \rightarrow \mathrm{C}_{6} \mathrm{H}_{5} \mathrm{OCH}_{3}+\mathrm{CH}_{3} \mathrm{OH}$ & 70.9 & -258.4 \\
\hline 5 & $1,4-\mathrm{C}_{6} \mathrm{H}_{4}(\mathrm{OH})_{2}+\mathrm{CH}_{3} \mathrm{CH}_{2} \mathrm{CH}_{3} \rightarrow 2,3-\left(\mathrm{CH}_{3}\right)_{2} \mathrm{C}_{6} \mathrm{H}_{3}(\mathrm{OH})+\mathrm{CH}_{3} \mathrm{OH}$ & 4.3 & -257.8 \\
\hline 6 & $1,4-\mathrm{C}_{6} \mathrm{H}_{4}(\mathrm{OH})_{2}+\mathrm{CH}_{3} \mathrm{CH}_{2} \mathrm{CH}_{3} \rightarrow 2,5-\left(\mathrm{CH}_{3}\right)_{2} \mathrm{C}_{6} \mathrm{H}_{3}(\mathrm{OH})+\mathrm{CH}_{3} \mathrm{OH}$ & 1.4 & -259.3 \\
\hline 7 & $1,4-\mathrm{C}_{6} \mathrm{H}_{4}(\mathrm{OH})_{2}+\mathrm{CH}_{3} \mathrm{CH}_{2} \mathrm{CH}_{3} \rightarrow 2,6-\left(\mathrm{CH}_{3}\right)_{2} \mathrm{C}_{6} \mathrm{H}_{3}(\mathrm{OH})+\mathrm{CH}_{3} \mathrm{OH}$ & -0.2 & -258.2 \\
\hline 8 & $1,4-\mathrm{C}_{6} \mathrm{H}_{4}(\mathrm{OH})_{2}+\mathrm{CH}_{3} \mathrm{CH}_{2} \mathrm{CH}_{3} \rightarrow 3,4-\left(\mathrm{CH}_{3}\right)_{2} \mathrm{C}_{6} \mathrm{H}_{3}(\mathrm{OH})+\mathrm{CH}_{3} \mathrm{OH}$ & 4.7 & -258.3 \\
\hline 9 & $1,4-\mathrm{C}_{6} \mathrm{H}_{4}(\mathrm{OH})_{2}+\mathrm{CH}_{3} \mathrm{CH}_{2} \mathrm{CH}_{2} \mathrm{CH}_{3} \rightarrow 2,3,6-\left(\mathrm{CH}_{3}\right)_{3} \mathrm{C}_{6} \mathrm{H}_{2}(\mathrm{OH})+\mathrm{CH}_{3} \mathrm{OH}$ & -8.6 & -259.2 \\
\hline 10 & $1,4-\mathrm{C}_{6} \mathrm{H}_{4}(\mathrm{OH})_{2}+\mathrm{CH}_{3} \mathrm{CH}_{3} \rightarrow \mathrm{C}_{6} \mathrm{H}_{6}+\mathrm{HOCH}_{2} \mathrm{CH}_{2} \mathrm{OH}$ & 37.9 & -260.5 \\
\hline 11 & $1,4-\mathrm{C}_{6} \mathrm{H}_{4}(\mathrm{OH})_{2}+\mathrm{CH}_{4} \rightarrow \mathrm{C}_{6} \mathrm{H}_{6}+\mathrm{CH}_{2}(\mathrm{OH})_{2}$ & 25.6 & -260.8 \\
\hline 12 & $1,4-\mathrm{C}_{6} \mathrm{H}_{4}(\mathrm{OH})_{2}+\mathrm{CH}_{3} \mathrm{CH}_{2} \mathrm{CH}_{3} \rightarrow \mathrm{C}_{6} \mathrm{H}_{6}+\mathrm{HOCH}_{2} \mathrm{CH}_{2} \mathrm{CH}_{2} \mathrm{OH}$ & 37.7 & -261.0 \\
\hline 13 & $1,4-\mathrm{C}_{6} \mathrm{H}_{4}(\mathrm{OH})_{2}+\mathrm{CH}_{3} \mathrm{CH}_{2} \mathrm{CH}_{3} \rightarrow \mathrm{C}_{6} \mathrm{H}_{6}+\mathrm{HOCH}_{2} \mathrm{CH}(\mathrm{OH}) \mathrm{CH}_{3}$ & 18.3 & -259.4 \\
\hline 14 & $1,4-\mathrm{C}_{6} \mathrm{H}_{4}(\mathrm{OH})_{2} \rightarrow 1,2-\mathrm{C}_{6} \mathrm{H}_{4}(\mathrm{OH})_{2}$ & -9.9 & -259.2 \\
\hline 15 & $1,4-\mathrm{C}_{6} \mathrm{H}_{4}(\mathrm{OH})_{2} \rightarrow 1,3-\mathrm{C}_{6} \mathrm{H}_{4}(\mathrm{OH})_{2}$ & -8.7 & -259.1 \\
\hline & & average: & $-259.3 \pm 3.0$ \\
\hline \multicolumn{4}{|c|}{ 1,2,3-trihydroxybenzene } \\
\hline 1 & $1,2,3-\mathrm{C}_{6} \mathrm{H}_{3}(\mathrm{OH})_{3}+2 \mathrm{C}_{6} \mathrm{H}_{6} \rightarrow 3 \mathrm{C}_{6} \mathrm{H}_{5} \mathrm{OH}$ & 4.5 & -445.7 \\
\hline 2 & $1,2,3-\mathrm{C}_{6} \mathrm{H}_{3}(\mathrm{OH})_{3}+3 \mathrm{CH}_{4} \rightarrow \mathrm{C}_{6} \mathrm{H}_{6} \rightarrow 3 \mathrm{CH}_{3} \mathrm{OH}$ & 151.9 & -447.6 \\
\hline 3 & $1,2,3-\mathrm{C}_{6} \mathrm{H}_{3}(\mathrm{OH})_{3}+\mathrm{CH}_{4}+\mathrm{CH}_{3} \mathrm{CH}_{3} \rightarrow 1,2-\left(\mathrm{CH}_{3}\right) \mathrm{C}_{6} \mathrm{H}_{4}(\mathrm{OH})+2 \mathrm{CH}_{3} \mathrm{OH}$ & 75.2 & -446.8 \\
\hline 4 & $1,2,3-\mathrm{C}_{6} \mathrm{H}_{3}(\mathrm{OH})_{3}+\mathrm{CH}_{4}+\mathrm{CH}_{3} \mathrm{CH}_{3} \rightarrow \mathrm{C}_{6} \mathrm{H}_{5} \mathrm{OCH}_{3}+2 \mathrm{CH}_{3} \mathrm{OH}$ & 132.3 & -446.0 \\
\hline 5 & $1,2,3-\mathrm{C}_{6} \mathrm{H}_{3}(\mathrm{OH})_{3}+\mathrm{CH}_{4}+\mathrm{CH}_{3} \mathrm{CH}_{2} \mathrm{CH}_{3} \rightarrow 2,3-\left(\mathrm{CH}_{3}\right)_{2} \mathrm{C}_{6} \mathrm{H}_{3}(\mathrm{OH})+2 \mathrm{CH}_{3} \mathrm{OH}$ & 65.8 & -445.5 \\
\hline 6 & $1,2,3-\mathrm{C}_{6} \mathrm{H}_{3}(\mathrm{OH})_{3}+\mathrm{CH}_{4}+\mathrm{CH}_{3} \mathrm{CH}_{2} \mathrm{CH}_{3} \rightarrow 2,5-\left(\mathrm{CH}_{3}\right)_{2} \mathrm{C}_{6} \mathrm{H}_{3}(\mathrm{OH})+2 \mathrm{CH}_{3} \mathrm{OH}$ & 62.9 & -447.0 \\
\hline 7 & $1,2,3-\mathrm{C}_{6} \mathrm{H}_{3}(\mathrm{OH})_{3}+\mathrm{CH}_{4}+\mathrm{CH}_{3} \mathrm{CH}_{2} \mathrm{CH}_{3} \rightarrow 2,6-\left(\mathrm{CH}_{3}\right)_{2} \mathrm{C}_{6} \mathrm{H}_{3}(\mathrm{OH})+2 \mathrm{CH}_{3} \mathrm{OH}$ & 61.2 & -445.8 \\
\hline 8 & $1,2,3-\mathrm{C}_{6} \mathrm{H}_{3}(\mathrm{OH})_{3}+\mathrm{CH}_{4}+\mathrm{CH}_{3} \mathrm{CH}_{2} \mathrm{CH}_{3} \rightarrow 3,4-\left(\mathrm{CH}_{3}\right)_{2} \mathrm{C}_{6} \mathrm{H}_{3}(\mathrm{OH})+2 \mathrm{CH}_{3} \mathrm{OH}$ & 66.1 & -445.9 \\
\hline 9 & $1,2,3-\mathrm{C}_{6} \mathrm{H}_{3}(\mathrm{OH})_{3}+\mathrm{CH}_{4}+\mathrm{CH}_{3} \mathrm{CH}_{2} \mathrm{CH}_{2} \mathrm{CH}_{3} \rightarrow 2,3,6-\left(\mathrm{CH}_{3}\right)_{3} \mathrm{C}_{6} \mathrm{H}_{2}(\mathrm{OH})+2 \mathrm{CH}_{3} \mathrm{OH}$ & 52.9 & -446.8 \\
\hline 10 & $1,2,3-\mathrm{C}_{6} \mathrm{H}_{3}(\mathrm{OH})_{3}+\mathrm{CH}_{4}+\mathrm{CH}_{3} \mathrm{CH}_{3} \rightarrow \mathrm{C}_{6} \mathrm{H}_{6}+\mathrm{HOCH}_{2} \mathrm{CH}_{2} \mathrm{OH}+\mathrm{CH}_{3} \mathrm{OH}$ & 99.4 & -448.2 \\
\hline 11 & $1,2,3-\mathrm{C}_{6} \mathrm{H}_{3}(\mathrm{OH})_{3}+2 \mathrm{CH}_{4} \rightarrow \mathrm{C}_{6} \mathrm{H}_{6}+\mathrm{CH}_{2}(\mathrm{OH})_{2}+\mathrm{CH}_{3} \mathrm{OH}$ & 87.1 & -448.5 \\
\hline 12 & $1,2,3-\mathrm{C}_{6} \mathrm{H}_{3}(\mathrm{OH})_{3}+\mathrm{CH}_{4}+\mathrm{CH}_{3} \mathrm{CH}_{2} \mathrm{CH}_{3} \rightarrow \mathrm{C}_{6} \mathrm{H}_{6}+\mathrm{HOCH}_{2} \mathrm{CH}_{2} \mathrm{CH}_{2} \mathrm{OH}+\mathrm{CH}_{3} \mathrm{OH}$ & 99.1 & -448.6 \\
\hline 13 & $1,2,3-\mathrm{C}_{6} \mathrm{H}_{3}(\mathrm{OH})_{3}+\mathrm{CH}_{4}+\mathrm{CH}_{3} \mathrm{CH}_{2} \mathrm{CH}_{3} \rightarrow \mathrm{C}_{6} \mathrm{H}_{6}+\mathrm{HOCH}_{2} \mathrm{CH}(\mathrm{OH}) \mathrm{CH}_{3}+\mathrm{CH}_{3} \mathrm{OH}$ & 79.7 & -447.0 \\
\hline 14 & $1,2,3-\mathrm{C}_{6} \mathrm{H}_{3}(\mathrm{OH})_{3}+\mathrm{CH}_{3} \mathrm{CH}_{2} \mathrm{CH}_{3} \rightarrow \mathrm{C}_{6} \mathrm{H}_{6}+\mathrm{HOCH}_{2} \mathrm{CH}(\mathrm{OH}) \mathrm{CH}_{2} \mathrm{OH}$ & 52.4 & -448.4 \\
\hline
\end{tabular}




\begin{tabular}{|c|c|c|c|}
\hline 15 & $1,2,3-\mathrm{C}_{6} \mathrm{H}_{3}(\mathrm{OH})_{3}+\mathrm{C}_{6} \mathrm{H}_{6} \rightarrow 1,2-\mathrm{C}_{6} \mathrm{H}_{4}(\mathrm{OH})_{2}+\mathrm{C}_{6} \mathrm{H}_{5} \mathrm{OH}$ & 2.3 & -446.3 \\
\hline 16 & $1,2,3-\mathrm{C}_{6} \mathrm{H}_{3}(\mathrm{OH})_{3}+\mathrm{C}_{6} \mathrm{H}_{6} \rightarrow 1,3-\mathrm{C}_{6} \mathrm{H}_{4}(\mathrm{OH})_{2}+\mathrm{C}_{6} \mathrm{H}_{5} \mathrm{OH}$ & 3.6 & -446.3 \\
\hline 17 & $1,2,3-\mathrm{C}_{6} \mathrm{H}_{3}(\mathrm{OH})_{3}+\mathrm{C}_{6} \mathrm{H}_{6} \rightarrow 1,4-\mathrm{C}_{6} \mathrm{H}_{4}(\mathrm{OH})_{2}+\mathrm{C}_{6} \mathrm{H}_{5} \mathrm{OH}$ & 12.3 & -446.3 \\
\hline \multicolumn{4}{|c|}{ 1,2,4-trihydroxybenzene } \\
\hline 1 & $1,2,4-\mathrm{C}_{6} \mathrm{H}_{3}(\mathrm{OH})_{3}+2 \mathrm{C}_{6} \mathrm{H}_{6} \rightarrow 3 \mathrm{C}_{6} \mathrm{H}_{5} \mathrm{OH}$ & -4.4 & -436.8 \\
\hline 2 & $1,2,4-\mathrm{C}_{6} \mathrm{H}_{3}(\mathrm{OH})_{3}+3 \mathrm{CH}_{4} \rightarrow \mathrm{C}_{6} \mathrm{H}_{6} \rightarrow 3 \mathrm{CH}_{3} \mathrm{OH}$ & 143.1 & -438.8 \\
\hline 3 & $1,2,4-\mathrm{C}_{6} \mathrm{H}_{3}(\mathrm{OH})_{3}+\mathrm{CH}_{4}+\mathrm{CH}_{3} \mathrm{CH}_{3} \rightarrow 1,2-\left(\mathrm{CH}_{3}\right) \mathrm{C}_{6} \mathrm{H}_{4}(\mathrm{OH})+2 \mathrm{CH}_{3} \mathrm{OH}$ & 66.3 & -437.9 \\
\hline 4 & $1,2,4-\mathrm{C}_{6} \mathrm{H}_{3}(\mathrm{OH})_{3}+\mathrm{CH}_{4}+\mathrm{CH}_{3} \mathrm{CH}_{3} \rightarrow \mathrm{C}_{6} \mathrm{H}_{5} \mathrm{OCH}_{3}+2 \mathrm{CH}_{3} \mathrm{OH}$ & 123.5 & -437.2 \\
\hline 5 & $1,2,4-\mathrm{C}_{6} \mathrm{H}_{3}(\mathrm{OH})_{3}+\mathrm{CH}_{4}+\mathrm{CH}_{3} \mathrm{CH}_{2} \mathrm{CH}_{3} \rightarrow 2,3-\left(\mathrm{CH}_{3}\right)_{2} \mathrm{C}_{6} \mathrm{H}_{3}(\mathrm{OH})+2 \mathrm{CH}_{3} \mathrm{OH}$ & 56.9 & -436.6 \\
\hline 6 & $1,2,4-\mathrm{C}_{6} \mathrm{H}_{3}(\mathrm{OH})_{3}+\mathrm{CH}_{4}+\mathrm{CH}_{3} \mathrm{CH}_{2} \mathrm{CH}_{3} \rightarrow 2,5-\left(\mathrm{CH}_{3}\right)_{2} \mathrm{C}_{6} \mathrm{H}_{3}(\mathrm{OH})+2 \mathrm{CH}_{3} \mathrm{OH}$ & 54.0 & -438.1 \\
\hline 7 & $1,2,4-\mathrm{C}_{6} \mathrm{H}_{3}(\mathrm{OH})_{3}+\mathrm{CH}_{4}+\mathrm{CH}_{3} \mathrm{CH}_{2} \mathrm{CH}_{3} \rightarrow 2,6-\left(\mathrm{CH}_{3}\right)_{2} \mathrm{C}_{6} \mathrm{H}_{3}(\mathrm{OH})+2 \mathrm{CH}_{3} \mathrm{OH}$ & 52.4 & -437.0 \\
\hline 8 & $1,2,4-\mathrm{C}_{6} \mathrm{H}_{3}(\mathrm{OH})_{3}+\mathrm{CH}_{4}+\mathrm{CH}_{3} \mathrm{CH}_{2} \mathrm{CH}_{3} \rightarrow 3,4-\left(\mathrm{CH}_{3}\right)_{2} \mathrm{C}_{6} \mathrm{H}_{3}(\mathrm{OH})+2 \mathrm{CH}_{3} \mathrm{OH}$ & 57.3 & -437.1 \\
\hline 9 & $1,2,4-\mathrm{C}_{6} \mathrm{H}_{3}(\mathrm{OH})_{3}+\mathrm{CH}_{4}+\mathrm{CH}_{3} \mathrm{CH}_{2} \mathrm{CH}_{2} \mathrm{CH}_{3} \rightarrow 2,3,6-\left(\mathrm{CH}_{3}\right)_{3} \mathrm{C}_{6} \mathrm{H}_{2}(\mathrm{OH})+2 \mathrm{CH}_{3} \mathrm{OH}$ & 44.0 & -437.9 \\
\hline 10 & $1,2,4-\mathrm{C}_{6} \mathrm{H}_{3}(\mathrm{OH})_{3}+\mathrm{CH}_{4}+\mathrm{CH}_{3} \mathrm{CH}_{3} \rightarrow \mathrm{C}_{6} \mathrm{H}_{6}+\mathrm{HOCH}_{2} \mathrm{CH}_{2} \mathrm{OH}+\mathrm{CH}_{3} \mathrm{OH}$ & 90.5 & -439.3 \\
\hline 11 & $1,2,4-\mathrm{C}_{6} \mathrm{H}_{3}(\mathrm{OH})_{3}+2 \mathrm{CH}_{4} \rightarrow \mathrm{C}_{6} \mathrm{H}_{6}+\mathrm{CH}_{2}(\mathrm{OH})_{2}+\mathrm{CH}_{3} \mathrm{OH}$ & 78.2 & -439.6 \\
\hline 12 & $1,2,4-\mathrm{C}_{6} \mathrm{H}_{3}(\mathrm{OH})_{3}+\mathrm{CH}_{4}+\mathrm{CH}_{3} \mathrm{CH}_{2} \mathrm{CH}_{3} \rightarrow \mathrm{C}_{6} \mathrm{H}_{6}+\mathrm{HOCH}_{2} \mathrm{CH}_{2} \mathrm{CH}_{2} \mathrm{OH}+\mathrm{CH}_{3} \mathrm{OH}$ & 90.3 & -439.8 \\
\hline 13 & $1,2,4-\mathrm{C}_{6} \mathrm{H}_{3}(\mathrm{OH})_{3}+\mathrm{CH}_{4}+\mathrm{CH}_{3} \mathrm{CH}_{2} \mathrm{CH}_{3} \rightarrow \mathrm{C}_{6} \mathrm{H}_{6}+\mathrm{HOCH}_{2} \mathrm{CH}(\mathrm{OH}) \mathrm{CH}_{3}+\mathrm{CH}_{3} \mathrm{OH}$ & 70.9 & -438.2 \\
\hline 14 & $1,2,4-\mathrm{C}_{6} \mathrm{H}_{3}(\mathrm{OH})_{3}+\mathrm{CH}_{3} \mathrm{CH}_{2} \mathrm{CH}_{3} \rightarrow \mathrm{C}_{6} \mathrm{H}_{6}+\mathrm{HOCH}_{2} \mathrm{CH}(\mathrm{OH}) \mathrm{CH}_{2} \mathrm{OH}$ & 43.6 & -439.6 \\
\hline 15 & $1,2,4-\mathrm{C}_{6} \mathrm{H}_{3}(\mathrm{OH})_{3}+\mathrm{C}_{6} \mathrm{H}_{6} \rightarrow 1,2-\mathrm{C}_{6} \mathrm{H}_{4}(\mathrm{OH})_{2}+\mathrm{C}_{6} \mathrm{H}_{5} \mathrm{OH}$ & -6.5 & -437.5 \\
\hline 16 & $1,2,4-\mathrm{C}_{6} \mathrm{H}_{3}(\mathrm{OH})_{3}+\mathrm{C}_{6} \mathrm{H}_{6} \rightarrow 1,3-\mathrm{C}_{6} \mathrm{H}_{4}(\mathrm{OH})_{2}+\mathrm{C}_{6} \mathrm{H}_{5} \mathrm{OH}$ & -5.2 & -437.5 \\
\hline 17 & $1,2,4-\mathrm{C}_{6} \mathrm{H}_{3}(\mathrm{OH})_{3}+\mathrm{C}_{6} \mathrm{H}_{6} \rightarrow 1,4-\mathrm{C}_{6} \mathrm{H}_{4}(\mathrm{OH})_{2}+\mathrm{C}_{6} \mathrm{H}_{5} \mathrm{OH}$ & 3.4 & -437.4 \\
\hline 18 & $1,2,4-\mathrm{C}_{6} \mathrm{H}_{3}(\mathrm{OH})_{3} \rightarrow 1,2,3-\mathrm{C}_{6} \mathrm{H}_{3}(\mathrm{OH})_{3}$ & -8.9 & -437.8 \\
\hline \multirow{2}{*}{\multicolumn{4}{|c|}{ 1,3,5-trihydroxybenzene }} \\
\hline & & & \\
\hline 1 & $1,3,5-\mathrm{C}_{6} \mathrm{H}_{3}(\mathrm{OH})_{3}+2 \mathrm{C}_{6} \mathrm{H}_{6} \rightarrow 3 \mathrm{C}_{6} \mathrm{H}_{5} \mathrm{OH}$ & 2.3 & -443.5 \\
\hline 2 & $1,3,5-\mathrm{C}_{6} \mathrm{H}_{3}(\mathrm{OH})_{3}+3 \mathrm{CH}_{4} \rightarrow \mathrm{C}_{6} \mathrm{H}_{6} \rightarrow 3 \mathrm{CH}_{3} \mathrm{OH}$ & 149.8 & -445.5 \\
\hline 3 & $1,3,5-\mathrm{C}_{6} \mathrm{H}_{3}(\mathrm{OH})_{3}+\mathrm{CH}_{4}+\mathrm{CH}_{3} \mathrm{CH}_{3} \rightarrow 1,2-\left(\mathrm{CH}_{3}\right) \mathrm{C}_{6} \mathrm{H}_{4}(\mathrm{OH})+2 \mathrm{CH}_{3} \mathrm{OH}$ & 73.0 & -444.6 \\
\hline 4 & $1,3,5-\mathrm{C}_{6} \mathrm{H}_{3}(\mathrm{OH})_{3}+\mathrm{CH}_{4}+\mathrm{CH}_{3} \mathrm{CH}_{3} \rightarrow \mathrm{C}_{6} \mathrm{H}_{5} \mathrm{OCH}_{3}+2 \mathrm{CH}_{3} \mathrm{OH}$ & 130.2 & -443.9 \\
\hline 5 & $1,3,5-\mathrm{C}_{6} \mathrm{H}_{3}(\mathrm{OH})_{3}+\mathrm{CH}_{4}+\mathrm{CH}_{3} \mathrm{CH}_{2} \mathrm{CH}_{3} \rightarrow 2,3-\left(\mathrm{CH}_{3}\right)_{2} \mathrm{C}_{6} \mathrm{H}_{3}(\mathrm{OH})+2 \mathrm{CH}_{3} \mathrm{OH}$ & 63.6 & -443.3 \\
\hline 6 & $1,3,5-\mathrm{C}_{6} \mathrm{H}_{3}(\mathrm{OH})_{3}+\mathrm{CH}_{4}+\mathrm{CH}_{3} \mathrm{CH}_{2} \mathrm{CH}_{3} \rightarrow 2,5-\left(\mathrm{CH}_{3}\right)_{2} \mathrm{C}_{6} \mathrm{H}_{3}(\mathrm{OH})+2 \mathrm{CH}_{3} \mathrm{OH}$ & 60.7 & -444.8 \\
\hline 7 & $1,3,5-\mathrm{C}_{6} \mathrm{H}_{3}(\mathrm{OH})_{3}+\mathrm{CH}_{4}+\mathrm{CH}_{3} \mathrm{CH}_{2} \mathrm{CH}_{3} \rightarrow 2,6-\left(\mathrm{CH}_{3}\right)_{2} \mathrm{C}_{6} \mathrm{H}_{3}(\mathrm{OH})+2 \mathrm{CH}_{3} \mathrm{OH}$ & 59.0 & -443.6 \\
\hline 8 & $1,3,5-\mathrm{C}_{6} \mathrm{H}_{3}(\mathrm{OH})_{3}+\mathrm{CH}_{4}+\mathrm{CH}_{3} \mathrm{CH}_{2} \mathrm{CH}_{3} \rightarrow 3,4-\left(\mathrm{CH}_{3}\right)_{2} \mathrm{C}_{6} \mathrm{H}_{3}(\mathrm{OH})+2 \mathrm{CH}_{3} \mathrm{OH}$ & 63.9 & -443.7 \\
\hline 9 & $1,3,5-\mathrm{C}_{6} \mathrm{H}_{3}(\mathrm{OH})_{3}+\mathrm{CH}_{4}+\mathrm{CH}_{3} \mathrm{CH}_{2} \mathrm{CH}_{2} \mathrm{CH}_{3} \rightarrow 2,3,6-\left(\mathrm{CH}_{3}\right)_{3} \mathrm{C}_{6} \mathrm{H}_{2}(\mathrm{OH})+2 \mathrm{CH}_{3} \mathrm{OH}$ & 50.7 & -444.6 \\
\hline 10 & $1,3,5-\mathrm{C}_{6} \mathrm{H}_{3}(\mathrm{OH})_{3}+\mathrm{CH}_{4}+\mathrm{CH}_{3} \mathrm{CH}_{3} \rightarrow \mathrm{C}_{6} \mathrm{H}_{6}+\mathrm{HOCH}_{2} \mathrm{CH}_{2} \mathrm{OH}+\mathrm{CH}_{3} \mathrm{OH}$ & 97.2 & -446.0 \\
\hline 11 & $1,3,5-\mathrm{C}_{6} \mathrm{H}_{3}(\mathrm{OH})_{3}+2 \mathrm{CH}_{4} \rightarrow \mathrm{C}_{6} \mathrm{H}_{6}+\mathrm{CH}_{2}(\mathrm{OH})_{2}+\mathrm{CH}_{3} \mathrm{OH}$ & 84.9 & -446.3 \\
\hline 12 & $1,3,5-\mathrm{C}_{6} \mathrm{H}_{3}(\mathrm{OH})_{3}+\mathrm{CH}_{4}+\mathrm{CH}_{3} \mathrm{CH}_{2} \mathrm{CH}_{3} \rightarrow \mathrm{C}_{6} \mathrm{H}_{6}+\mathrm{HOCH}_{2} \mathrm{CH}_{2} \mathrm{CH}_{2} \mathrm{OH}+\mathrm{CH}_{3} \mathrm{OH}$ & 97.0 & -446.5 \\
\hline 13 & $1,3,5-\mathrm{C}_{6} \mathrm{H}_{3}(\mathrm{OH})_{3}+\mathrm{CH}_{4}+\mathrm{CH}_{3} \mathrm{CH}_{2} \mathrm{CH}_{3} \rightarrow \mathrm{C}_{6} \mathrm{H}_{6}+\mathrm{HOCH}_{2} \mathrm{CH}(\mathrm{OH}) \mathrm{CH}_{3}+\mathrm{CH}_{3} \mathrm{OH}$ & 77.6 & -444.9 \\
\hline 14 & $1,3,5-\mathrm{C}_{6} \mathrm{H}_{3}(\mathrm{OH})_{3}+\mathrm{CH}_{3} \mathrm{CH}_{2} \mathrm{CH}_{3} \rightarrow \mathrm{C}_{6} \mathrm{H}_{6}+\mathrm{HOCH}_{2} \mathrm{CH}(\mathrm{OH}) \mathrm{CH}_{2} \mathrm{OH}$ & 50.2 & -446.2 \\
\hline 15 & $1,3,5-\mathrm{C}_{6} \mathrm{H}_{3}(\mathrm{OH})_{3}+\mathrm{C}_{6} \mathrm{H}_{6} \rightarrow 1,2-\mathrm{C}_{6} \mathrm{H}_{4}(\mathrm{OH})_{2}+\mathrm{C}_{6} \mathrm{H}_{5} \mathrm{OH}$ & 0.2 & -444.2 \\
\hline 16 & $1,3,5-\mathrm{C}_{6} \mathrm{H}_{3}(\mathrm{OH})_{3}+\mathrm{C}_{6} \mathrm{H}_{6} \rightarrow 1,3-\mathrm{C}_{6} \mathrm{H}_{4}(\mathrm{OH})_{2}+\mathrm{C}_{6} \mathrm{H}_{5} \mathrm{OH}$ & 1.4 & -444.1 \\
\hline 17 & $1,3,5-\mathrm{C}_{6} \mathrm{H}_{3}(\mathrm{OH})_{3}+\mathrm{C}_{6} \mathrm{H}_{6} \rightarrow 1,4-\mathrm{C}_{6} \mathrm{H}_{4}(\mathrm{OH})_{2}+\mathrm{C}_{6} \mathrm{H}_{5} \mathrm{OH}$ & 10.1 & -444.1 \\
\hline 18 & $1,3,5-\mathrm{C}_{6} \mathrm{H}_{3}(\mathrm{OH})_{3} \rightarrow 1,2,3-\mathrm{C}_{6} \mathrm{H}_{3}(\mathrm{OH})_{3}$ & -2.2 & -444.5 \\
\hline \multirow[t]{2}{*}{19} & $1,3,5-\mathrm{C}_{6} \mathrm{H}_{3}(\mathrm{OH})_{3} \rightarrow 1,2,4-\mathrm{C}_{6} \mathrm{H}_{3}(\mathrm{OH})_{3}$ & 6.7 & -444.5 \\
\hline & & average: & $-444.7 \pm 3.0$ \\
\hline
\end{tabular}

${ }^{a}$ Experimental enthalpies of formation of all species used in isodesmic reactions are given in Table S1. The sets of isodesmic reactions for each compound include one reaction with phenol (given in red) for which the theoretical value of enthalpy of formation, $-91.8 \mathrm{~kJ} / \mathrm{mol}$, was used. ${ }^{b}$ The values of enthalpies of reaction $\left(\Delta_{\mathrm{r}} H_{298}^{\circ}\right)$ are given together with enthalpies of formation $\left(\Delta_{\mathrm{f}} H_{298}^{\circ}\right)$ for each isodesmic reaction. 
Table S4. Enthalpies of Formation of Gaseous $\mathrm{C}_{6} \mathrm{H}_{5} \mathrm{O}, \mathrm{C}_{6} \mathrm{H}_{5}$, and $\mathrm{CH}_{3} \mathrm{O}$ Radicals Calculated from Atomization Reaction and Isogyric Reactions Using G4 Energies

\begin{tabular}{|c|c|c|c|}
\hline \multicolumn{2}{|c|}{ reaction $^{a}$} & \multirow{2}{*}{$\begin{array}{c}\Delta_{\mathrm{r}} H_{298}^{\circ} \\
\mathrm{kJ} / \mathrm{mol}\end{array}$} & \multirow{2}{*}{$\begin{array}{l}\Delta_{\mathrm{f}} H_{298}^{\circ}{ }^{b} \\
\mathrm{~kJ} / \mathrm{mol}\end{array}$} \\
\hline \multirow{2}{*}{\multicolumn{4}{|c|}{$\mathrm{C}_{6} \mathrm{H}_{5} \mathrm{O} \bullet$}} \\
\hline atomization reaction & & & \\
\hline & $\mathrm{C}_{6} \mathrm{H}_{5} \mathrm{O} \cdot \rightarrow 6 \mathrm{C}(\mathrm{cr})+5 / 2 \mathrm{H}_{2}(\mathrm{~g})+1 / 2 \mathrm{O}_{2}(\mathrm{~g})$ & & 58.6 \\
\hline \multicolumn{4}{|c|}{ isogyric reactions } \\
\hline 1 & $\mathrm{C}_{6} \mathrm{H}_{5} \mathrm{O} \bullet+\mathrm{CH}_{4} \rightarrow \mathrm{CH}_{3} \mathrm{O} \bullet+\mathrm{C}_{6} \mathrm{H}_{6}$ & 118.5 & 60.8 \\
\hline 2 & $\mathrm{C}_{6} \mathrm{H}_{5} \mathrm{O} \bullet+\mathrm{CH}_{3} \mathrm{CH}_{3} \rightarrow \mathrm{CH}_{3} \mathrm{O} \bullet+\mathrm{C}_{6} \mathrm{H}_{5} \mathrm{CH}_{3}$ & 93.8 & 62.0 \\
\hline 3 & $\mathrm{C}_{6} \mathrm{H}_{5} \mathrm{O} \bullet+\mathrm{CH}_{3} \mathrm{OCH}_{3} \rightarrow \mathrm{CH}_{3} \mathrm{O} \bullet+\mathrm{C}_{6} \mathrm{H}_{5} \mathrm{OCH}_{3}$ & 74.1 & 61.1 \\
\hline 4 & $\mathrm{C}_{6} \mathrm{H}_{5} \mathrm{O} \bullet+\mathrm{CH}_{4} \rightarrow \mathrm{HOCH}_{2} \bullet+\mathrm{C}_{6} \mathrm{H}_{6}$ & 83.3 & 58.0 \\
\hline 5 & $\mathrm{C}_{6} \mathrm{H}_{5} \mathrm{O} \bullet+\mathrm{CH}_{3} \mathrm{CH}_{3} \rightarrow \mathrm{HOCH}_{2} \bullet+\mathrm{C}_{6} \mathrm{H}_{5} \mathrm{CH}_{3}$ & 58.7 & 59.2 \\
\hline 6 & $\mathrm{C}_{6} \mathrm{H}_{5} \mathrm{O} \bullet+\mathrm{CH}_{3} \mathrm{OCH}_{3} \rightarrow \mathrm{HOCH}_{2} \bullet+\mathrm{C}_{6} \mathrm{H}_{5} \mathrm{OCH}_{3}$ & 38.9 & 58.3 \\
\hline 7 & $\mathrm{C}_{6} \mathrm{H}_{5} \mathrm{O} \bullet+\mathrm{CH}_{3} \mathrm{CH}_{3} \rightarrow \mathrm{CH}_{3} \mathrm{CH}_{2} \mathrm{O} \bullet+\mathrm{C}_{6} \mathrm{H}_{6}$ & 93.5 & 61.4 \\
\hline 8 & $\mathrm{C}_{6} \mathrm{H}_{5} \mathrm{O} \bullet+\mathrm{CH}_{3} \mathrm{CH}_{2} \mathrm{CH}_{3} \rightarrow \mathrm{CH}_{3} \mathrm{CH}_{2} \mathrm{O} \bullet+\mathrm{C}_{6} \mathrm{H}_{5} \mathrm{CH}_{3}$ & 80.4 & 62.1 \\
\hline 9 & $\mathrm{C}_{6} \mathrm{H}_{5} \mathrm{O} \bullet+\mathrm{CH}_{3} \mathrm{CH}_{3} \rightarrow \mathrm{HOCH}_{2} \mathrm{CH}_{2} \bullet+\mathrm{C}_{6} \mathrm{H}_{6}$ & 81.4 & 59.6 \\
\hline 10 & $\mathrm{C}_{6} \mathrm{H}_{5} \mathrm{O} \bullet+\mathrm{CH}_{3} \mathrm{CH}_{2} \mathrm{CH}_{3} \rightarrow \mathrm{HOCH}_{2} \mathrm{CH}_{2} \bullet+\mathrm{C}_{6} \mathrm{H}_{5} \mathrm{CH}_{3}$ & 68.4 & 60.3 \\
\hline 11 & $\mathrm{C}_{6} \mathrm{H}_{5} \mathrm{O} \cdot+\mathrm{CH}_{4} \rightarrow \mathrm{HO} \cdot+\mathrm{C}_{6} \mathrm{H}_{5} \mathrm{CH}_{3}$ & 103.7 & 58.4 \\
\hline 12 & $\mathrm{C}_{6} \mathrm{H}_{5} \mathrm{O} \bullet+\mathrm{CH}_{3} \mathrm{CH}_{3} \rightarrow \mathrm{HO} \bullet+\mathrm{C}_{6} \mathrm{H}_{5} \mathrm{CH}_{2} \mathrm{CH}_{3}$ & 90.4 & 60.9 \\
\hline 13 & $\mathrm{C}_{6} \mathrm{H}_{5} \mathrm{O} \bullet+\mathrm{CH}_{3} \mathrm{CH}_{3} \rightarrow \mathrm{HO} \bullet+1,4-\mathrm{C}_{6} \mathrm{H}_{4}\left(\mathrm{CH}_{3}\right)_{2}$ & 80.2 & 59.2 \\
\hline 14 & $\mathrm{C}_{6} \mathrm{H}_{5} \mathrm{O} \bullet+\mathrm{CH}_{3} \mathrm{OOH} \rightarrow \mathrm{HOO} \bullet+\mathrm{C}_{6} \mathrm{H}_{5} \mathrm{OCH}_{3}$ & 11.1 & 58.3 \\
\hline 15 & $\mathrm{C}_{6} \mathrm{H}_{5} \mathrm{O} \cdot+\mathrm{H}_{2} \mathrm{O} \rightarrow \mathrm{HOO} \bullet+\mathrm{C}_{6} \mathrm{H}_{6}$ & 278.8 & 58.2 \\
\hline 16 & $\mathrm{C}_{6} \mathrm{H}_{5} \mathrm{O} \bullet+\mathrm{CH}_{4} \rightarrow \mathrm{C}_{6} \mathrm{H}_{5} \bullet+\mathrm{CH}_{3} \mathrm{OH}$ & 152.8 & 58.3 \\
\hline 17 & $\mathrm{C}_{6} \mathrm{H}_{5} \mathrm{O} \bullet+\mathrm{CH}_{3} \mathrm{CH}_{3} \rightarrow \mathrm{C}_{6} \mathrm{H}_{5} \bullet+\mathrm{CH}_{3} \mathrm{CH}_{2} \mathrm{OH}$ & 129.3 & 57.3 \\
\hline 18 & $\mathrm{C}_{6} \mathrm{H}_{5} \mathrm{O} \bullet+\mathrm{CH}_{3} \mathrm{CH}_{3} \rightarrow \mathrm{C}_{6} \mathrm{H}_{5} \bullet+\mathrm{CH}_{3} \mathrm{OCH}_{3}$ & 177.6 & 59.6 \\
\hline 19 & $\mathrm{C}_{6} \mathrm{H}_{5} \mathrm{O} \bullet+\mathrm{CH}_{3} \mathrm{CH}_{3} \rightarrow \mathrm{CH}_{3} \bullet+\mathrm{C}_{6} \mathrm{H}_{5} \mathrm{OCH}_{3}$ & 99.3 & 60.4 \\
\hline 20 & $\mathrm{C}_{6} \mathrm{H}_{5} \mathrm{O} \bullet+\mathrm{H}_{2} \mathrm{C}=\mathrm{NH} \rightarrow \mathrm{CH}_{3} \bullet+\mathrm{C}_{6} \mathrm{H}_{5} \mathrm{NO}$ & 195.8 & 60.6 \\
\hline 21 & $\mathrm{C}_{6} \mathrm{H}_{5} \mathrm{O} \bullet+\mathrm{CH}_{3} \mathrm{CH}_{2} \mathrm{CH}_{3} \rightarrow \mathrm{CH}_{3} \mathrm{CH}_{2} \bullet+\mathrm{C}_{6} \mathrm{H}_{5} \mathrm{OCH}_{3}$ & 95.0 & 58.6 \\
\hline 22 & $\mathrm{C}_{6} \mathrm{H}_{5} \mathrm{O} \bullet+\mathrm{H}_{2} \mathrm{C}=\mathrm{CHCH}_{3} \rightarrow \mathrm{H}_{2} \mathrm{C}=\mathrm{CH} \bullet+\mathrm{C}_{6} \mathrm{H}_{5} \mathrm{OCH}_{3}$ & 145.2 & 61.0 \\
\hline 23 & $\mathrm{C}_{6} \mathrm{H}_{5} \mathrm{O} \bullet+\mathrm{CH}_{3} \mathrm{C}(\mathrm{O}) \mathrm{H} \rightarrow \mathrm{HCO} \bullet+\mathrm{C}_{6} \mathrm{H}_{5} \mathrm{OCH}_{3}$ & 76.7 & 59.8 \\
\hline \multirow[t]{2}{*}{24} & $\mathrm{C}_{6} \mathrm{H}_{5} \mathrm{O} \bullet+\mathrm{CH}_{3} \mathrm{C}(\mathrm{O}) \mathrm{CH}_{3} \rightarrow \mathrm{CH}_{3} \mathrm{CO} \bullet+\mathrm{C}_{6} \mathrm{H}_{5} \mathrm{OCH}_{3}$ & 74.5 & 61.2 \\
\hline & \multicolumn{2}{|c|}{$\begin{array}{r}\mathrm{C}_{6} \mathrm{H}_{5} \mathrm{O} \bullet+\mathrm{CH}_{3} \mathrm{C}(\mathrm{O}) \mathrm{CH}_{3} \rightarrow \mathrm{CH}_{3} \mathrm{CO} \bullet+\mathrm{C}_{6} \mathrm{H}_{5} \mathrm{OCH}_{3} \\
\text { average of } 24 \text { reactions: }\end{array}$} & $\mathbf{5 9 . 8} \pm \mathbf{3 . 0}$ \\
\hline \multicolumn{4}{|c|}{$\mathrm{C}_{6} \mathrm{H}_{5}{ }^{\circ}$} \\
\hline \multicolumn{4}{|c|}{ atomization reaction } \\
\hline & $\mathrm{C}_{6} \mathrm{H}_{5} \bullet \rightarrow 6 \mathrm{C}(\mathrm{cr})+5 / 2 \mathrm{H}_{2}(\mathrm{~g})$ & & 337.9 \\
\hline \multicolumn{4}{|c|}{ isogyric reactions } \\
\hline 1 & $\mathrm{C}_{6} \mathrm{H}_{5} \bullet+\mathrm{CH}_{4} \rightarrow \mathrm{CH}_{3} \bullet+\mathrm{C}_{6} \mathrm{H}_{6}$ & -33.9 & 337.8 \\
\hline 2 & $\mathrm{C}_{6} \mathrm{H}_{5} \bullet+\mathrm{CH}_{3} \mathrm{CH}_{3} \rightarrow \mathrm{CH}_{3} \bullet+\mathrm{C}_{6} \mathrm{H}_{5} \mathrm{CH}_{3}$ & -58.5 & 339.0 \\
\hline 3 & $\mathrm{C}_{6} \mathrm{H}_{5} \bullet+\mathrm{CH}_{3} \mathrm{OCH}_{3} \rightarrow \mathrm{CH}_{3} \bullet+\mathrm{C}_{6} \mathrm{H}_{5} \mathrm{OCH}_{3}$ & -78.3 & 338.1 \\
\hline 4 & $\mathrm{C}_{6} \mathrm{H}_{5} \bullet+\mathrm{CH}_{3} \mathrm{CH}_{3} \rightarrow \mathrm{CH}_{3} \mathrm{CH}_{2} \bullet+\mathrm{C}_{6} \mathrm{H}_{6}$ & -49.8 & 336.5 \\
\hline 5 & $\mathrm{C}_{6} \mathrm{H}_{5} \bullet+\mathrm{CH}_{3} \mathrm{CH}_{2} \mathrm{CH}_{3} \rightarrow \mathrm{CH}_{3} \mathrm{CH}_{2} \bullet+\mathrm{C}_{6} \mathrm{H}_{5} \mathrm{CH}_{3}$ & -62.8 & 337.2 \\
\hline 6 & $\mathrm{C}_{6} \mathrm{H}_{5} \bullet+\mathrm{CH}_{3} \mathrm{CH}(\mathrm{OH}) \mathrm{CH}_{3} \rightarrow \mathrm{CH}_{3} \mathrm{CH}_{2} \bullet+\mathrm{C}_{6} \mathrm{H}_{5} \mathrm{OCH}_{3}$ & -15.7 & 337.7 \\
\hline 7 & $\mathrm{C}_{6} \mathrm{H}_{5} \bullet+\mathrm{HNO}_{2} \rightarrow \mathrm{HO} \bullet+\mathrm{C}_{6} \mathrm{H}_{5} \mathrm{NO}$ & -24.7 & 340.0 \\
\hline 8 & $\mathrm{C}_{6} \mathrm{H}_{5} \bullet+\mathrm{CH}_{3} \mathrm{OH} \rightarrow \mathrm{HO} \bullet+\mathrm{C}_{6} \mathrm{H}_{5} \mathrm{CH}_{3}$ & -49.1 & 337.4 \\
\hline 9 & $\mathrm{C}_{6} \mathrm{H}_{5} \bullet+\mathrm{H}_{2} \mathrm{O} \rightarrow \mathrm{HO} \bullet+\mathrm{C}_{6} \mathrm{H}_{6}$ & 23.2 & 339.0 \\
\hline 10 & $\mathrm{C}_{6} \mathrm{H}_{5} \bullet+\mathrm{CH}_{3} \mathrm{OH} \rightarrow \mathrm{CH}_{3} \mathrm{O} \bullet+\mathrm{C}_{6} \mathrm{H}_{6}$ & -34.3 & 339.8 \\
\hline 11 & $\mathrm{C}_{6} \mathrm{H}_{5} \bullet+\mathrm{CH}_{3} \mathrm{OCH}_{3} \rightarrow \mathrm{CH}_{3} \mathrm{O} \bullet+\mathrm{C}_{6} \mathrm{H}_{5} \mathrm{CH}_{3}$ & -83.8 & 339.7 \\
\hline 12 & $\mathrm{C}_{6} \mathrm{H}_{5} \bullet+\mathrm{CH}_{3} \mathrm{OH} \rightarrow \mathrm{HOCH}_{2} \bullet+\mathrm{C}_{6} \mathrm{H}_{6}$ & -69.5 & 337.0 \\
\hline 13 & $\mathrm{C}_{6} \mathrm{H}_{5} \bullet+\mathrm{CH}_{3} \mathrm{OCH}_{3} \rightarrow \mathrm{HOCH}_{2} \bullet+\mathrm{C}_{6} \mathrm{H}_{5} \mathrm{CH}_{3}$ & -118.9 & 336.9 \\
\hline 14 & $\mathrm{C}_{6} \mathrm{H}_{5} \bullet+\mathrm{CH}_{3} \mathrm{CH}_{2} \mathrm{OH} \rightarrow \mathrm{CH}_{3} \mathrm{CH}_{2} \mathrm{O} \bullet+\mathrm{C}_{6} \mathrm{H}_{6}$ & -35.9 & 341.4 \\
\hline 15 & $\mathrm{C}_{6} \mathrm{H}_{5}^{\bullet}+\mathrm{CH}_{3} \mathrm{CH}_{2} \mathrm{CH}_{2} \mathrm{OH} \rightarrow \mathrm{CH}_{3} \mathrm{CH}_{2} \mathrm{O} \bullet+\mathrm{C}_{6} \mathrm{H}_{5} \mathrm{CH}_{3}$ & -49.3 & 342.5 \\
\hline 16 & $\mathrm{C}_{6} \mathrm{H}_{5} \bullet+\mathrm{CH}_{3} \mathrm{CH}_{2} \mathrm{OH} \rightarrow \mathrm{HOCH}_{2} \mathrm{CH}_{2} \bullet+\mathrm{C}_{6} \mathrm{H}_{6}$ & -61.4 & 340.8 \\
\hline 17 & $\mathrm{C}_{6} \mathrm{H}_{5} \bullet+\mathrm{CH}_{3} \mathrm{CH}_{2} \mathrm{CH}_{2} \mathrm{OH} \rightarrow \mathrm{HOCH}_{2} \mathrm{CH}_{2} \bullet+\mathrm{C}_{6} \mathrm{H}_{5} \mathrm{CH}_{3}$ & -47.9 & 339.6 \\
\hline
\end{tabular}




\begin{tabular}{|c|c|c|c|}
\hline 18 & $\mathrm{C}_{6} \mathrm{H}_{5} \bullet+\mathrm{CH}_{3} \mathrm{OOH} \rightarrow \mathrm{HOO} \bullet+\mathrm{C}_{6} \mathrm{H}_{5} \mathrm{CH}_{3}$ & -146.7 & 336.9 \\
\hline 19 & $\mathrm{C}_{6} \mathrm{H}_{5} \bullet+\mathrm{CH}_{2}(\mathrm{OH})_{2} \rightarrow \mathrm{HOO} \bullet+\mathrm{C}_{6} \mathrm{H}_{5} \mathrm{CH}_{3}$ & 118.6 & 336.4 \\
\hline 20 & $\mathrm{C}_{6} \mathrm{H}_{5} \bullet+\mathrm{HOOH} \rightarrow \mathrm{HOO} \bullet+\mathrm{C}_{6} \mathrm{H}_{6}$ & -108.0 & 338.6 \\
\hline 21 & $\mathrm{C}_{6} \mathrm{H}_{5} \bullet+\mathrm{H}_{2} \mathrm{C}=\mathrm{CHCH}_{3} \rightarrow \mathrm{H}_{2} \mathrm{C}=\mathrm{CH} \bullet+\mathrm{C}_{6} \mathrm{H}_{5} \mathrm{CH}_{3}$ & -12.7 & 339.7 \\
\hline 22 & $\mathrm{C}_{6} \mathrm{H}_{5} \bullet+\mathrm{H}_{2} \mathrm{C}=\mathrm{CH}_{2} \rightarrow \mathrm{H}_{2} \mathrm{C}=\mathrm{CH} \bullet+\mathrm{C}_{6} \mathrm{H}_{6}$ & -11.0 & 338.7 \\
\hline 23 & $\mathrm{C}_{6} \mathrm{H}_{5} \bullet+\mathrm{HC}(\mathrm{O}) \mathrm{H} \rightarrow \mathrm{HCO} \bullet+\mathrm{C}_{6} \mathrm{H}_{6}$ & -102.1 & 336.0 \\
\hline 24 & $\mathrm{C}_{6} \mathrm{H}_{5} \bullet+\mathrm{CH}_{3} \mathrm{C}(\mathrm{O}) \mathrm{H} \rightarrow \mathrm{HCO} \bullet+\mathrm{C}_{6} \mathrm{H}_{5} \mathrm{CH}_{3}$ & -81.2 & 338.5 \\
\hline 25 & $\mathrm{C}_{6} \mathrm{H}_{5} \bullet+\mathrm{CH}_{3} \mathrm{C}(\mathrm{O}) \mathrm{H} \rightarrow \mathrm{CH}_{3} \mathrm{CO} \bullet+\mathrm{C}_{6} \mathrm{H}_{6}$ & -99.8 & 338.4 \\
\hline \multirow[t]{3}{*}{26} & $\mathrm{C}_{6} \mathrm{H}_{5} \bullet+\mathrm{CH}_{3} \mathrm{C}(\mathrm{O}) \mathrm{CH}_{3} \rightarrow \mathrm{CH}_{3} \mathrm{CO} \bullet+\mathrm{C}_{6} \mathrm{H}_{5} \mathrm{CH}_{3}$ & -83.4 & 339.9 \\
\hline & \multicolumn{2}{|c|}{ average of 26 reactions: } & $338.6 \pm 4.0$ \\
\hline & \multicolumn{2}{|c|}{ experiment (ATcT): } & $337.3 \pm 0.6$ \\
\hline \multicolumn{4}{|c|}{$\mathrm{CH}_{3} \mathrm{O} \bullet$} \\
\hline \multicolumn{4}{|c|}{ atomization reaction } \\
\hline & $\mathrm{CH}_{3} \mathrm{O} \bullet \rightarrow \mathrm{C}(\mathrm{cr})+3 / 2 \mathrm{H}_{2}(\mathrm{~g})+1 / 2 \mathrm{O}_{2}(\mathrm{~g})$ & & 17.7 \\
\hline \multicolumn{4}{|c|}{ isogyric reactions } \\
\hline 1 & $\mathrm{CH}_{3} \mathrm{O} \bullet+\mathrm{CH}_{4} \rightarrow \mathrm{CH}_{3} \bullet+\mathrm{CH}_{3} \mathrm{OH}$ & 0.5 & 19.8 \\
\hline 2 & $\mathrm{CH}_{3} \mathrm{O} \bullet+\mathrm{CH}_{3} \mathrm{CH}_{3} \rightarrow \mathrm{CH}_{3} \bullet+\mathrm{CH}_{3} \mathrm{OCH}_{3}$ & 25.3 & 21.1 \\
\hline 3 & $\mathrm{CH}_{3} \mathrm{O} \bullet+\mathrm{C}_{6} \mathrm{H}_{5} \mathrm{CH}_{3} \rightarrow \mathrm{CH}_{3} \bullet+\mathrm{C}_{6} \mathrm{H}_{5} \mathrm{OCH}_{3}$ & 5.5 & 20.2 \\
\hline 4 & $\mathrm{CH}_{3} \mathrm{O} \bullet+\mathrm{CH}_{3} \mathrm{CH}_{3} \rightarrow \mathrm{CH}_{3} \mathrm{CH}_{2} \bullet+\mathrm{CH}_{3} \mathrm{OH}$ & -15.4 & 18.5 \\
\hline 5 & $\mathrm{CH}_{3} \mathrm{O} \bullet+\mathrm{CH}_{3} \mathrm{CH}_{2} \mathrm{CH}_{3} \rightarrow \mathrm{CH}_{3} \mathrm{CH}_{2} \bullet+\mathrm{CH}_{3} \mathrm{OCH}_{3}$ & 20.9 & 19.4 \\
\hline 6 & $\mathrm{CH}_{3} \mathrm{O} \bullet+\mathrm{H}_{2} \mathrm{C}=\mathrm{CH}_{2} \rightarrow \mathrm{H}_{2} \mathrm{C}=\mathrm{CH} \bullet+\mathrm{CH}_{3} \mathrm{OH}$ & 23.3 & 20.7 \\
\hline 7 & $\mathrm{CH}_{3} \mathrm{O} \bullet+\mathrm{H}_{2} \mathrm{C}=\mathrm{CHCH}_{3} \rightarrow \mathrm{H}_{2} \mathrm{C}=\mathrm{CH} \bullet+\mathrm{CH}_{3} \mathrm{OCH}_{3}$ & 71.1 & 21.8 \\
\hline 8 & $\mathrm{CH}_{3} \mathrm{O} \bullet+\mathrm{C}_{6} \mathrm{H}_{5} \mathrm{CH}=\mathrm{CH}_{2} \rightarrow \mathrm{H}_{2} \mathrm{C}=\mathrm{CH} \bullet+\mathrm{C}_{6} \mathrm{H}_{5} \mathrm{OCH}_{3}$ & 57.6 & 21.0 \\
\hline 9 & $\mathrm{CH}_{3} \mathrm{O} \bullet+\mathrm{C}_{6} \mathrm{H}_{6} \rightarrow \mathrm{C}_{6} \mathrm{H}_{5} \bullet+\mathrm{CH}_{3} \mathrm{OH}$ & 34.3 & 19.4 \\
\hline 10 & $\mathrm{CH}_{3} \mathrm{O} \bullet+\mathrm{C}_{6} \mathrm{H}_{5} \mathrm{CH}_{3} \rightarrow \mathrm{C}_{6} \mathrm{H}_{5} \bullet+\mathrm{CH}_{3} \mathrm{OCH}_{3}$ & 83.8 & 19.4 \\
\hline 11 & $\mathrm{CH}_{3} \mathrm{O} \bullet \rightarrow \mathrm{HOCH}_{2} \bullet$ & -35.2 & 19.1 \\
\hline 12 & $\mathrm{CH}_{3} \mathrm{O} \bullet+\mathrm{CH}_{3} \mathrm{CH}_{3} \rightarrow \mathrm{CH}_{3} \mathrm{CH}_{2} \mathrm{O} \bullet+\mathrm{CH}_{4}$ & -25.0 & 22.5 \\
\hline 13 & $\mathrm{CH}_{3} \mathrm{O} \bullet+\mathrm{CH}_{3} \mathrm{CH}_{2} \mathrm{CH}_{3} \rightarrow \mathrm{CH}_{3} \mathrm{CH}_{2} \mathrm{O} \bullet+\mathrm{CH}_{3} \mathrm{CH}_{3}$ & -13.4 & 22.0 \\
\hline 14 & $\mathrm{CH}_{3} \mathrm{O} \bullet+\mathrm{CH}_{3} \mathrm{CH}_{3} \rightarrow \mathrm{HOCH}_{2} \mathrm{CH}_{2} \bullet+\mathrm{CH}_{4}$ & -37.1 & 20.7 \\
\hline 15 & $\mathrm{CH}_{3} \mathrm{O} \bullet+\mathrm{CH}_{3} \mathrm{CH}_{2} \mathrm{CH}_{3} \rightarrow \mathrm{HOCH}_{2} \mathrm{CH}_{2} \bullet+\mathrm{CH}_{3} \mathrm{CH}_{3}$ & -25.5 & 20.2 \\
\hline 16 & $\mathrm{CH}_{3} \mathrm{O} \bullet+\mathrm{CH}_{4} \rightarrow \mathrm{HO} \bullet+\mathrm{CH}_{3} \mathrm{CH}_{3}$ & 9.9 & 18.2 \\
\hline 17 & $\mathrm{CH}_{3} \mathrm{O} \bullet+\mathrm{CH}_{3} \mathrm{CH}_{3} \rightarrow \mathrm{HO} \bullet+\mathrm{CH}_{3} \mathrm{CH}_{2} \mathrm{CH}_{3}$ & -1.7 & 18.7 \\
\hline 18 & $\mathrm{CH}_{3} \mathrm{O} \bullet+\mathrm{C}_{6} \mathrm{H}_{6} \rightarrow \mathrm{HO} \bullet+\mathrm{C}_{6} \mathrm{H}_{5} \mathrm{CH}_{3}$ & -14.8 & 19.5 \\
\hline 19 & $\mathrm{CH}_{3} \mathrm{O} \bullet+\mathrm{H}_{2} \mathrm{O} \rightarrow \mathrm{HOO} \bullet+\mathrm{CH}_{4}$ & 160.3 & 19.3 \\
\hline 20 & $\mathrm{CH}_{3} \mathrm{O} \bullet+\mathrm{HC}(\mathrm{O}) \mathrm{H} \rightarrow \mathrm{HCO} \bullet+\mathrm{CH}_{3} \mathrm{OH}$ & -67.8 & 18.1 \\
\hline 21 & $\mathrm{CH}_{3} \mathrm{O} \bullet+\mathrm{CH}_{3} \mathrm{C}(\mathrm{O}) \mathrm{H} \rightarrow \mathrm{HCO} \bullet+\mathrm{CH}_{3} \mathrm{OCH}_{3}$ & 2.6 & 20.6 \\
\hline 22 & $\mathrm{CH}_{3} \mathrm{O} \bullet+\mathrm{CH}_{3} \mathrm{C}(\mathrm{O}) \mathrm{H} \rightarrow \mathrm{CH}_{3} \mathrm{CO} \bullet+\mathrm{CH}_{3} \mathrm{OH}$ & -65.5 & 20.5 \\
\hline \multirow[t]{3}{*}{23} & $\mathrm{CH}_{3} \mathrm{O} \bullet+\mathrm{CH}_{3} \mathrm{C}(\mathrm{O}) \mathrm{CH}_{3} \rightarrow \mathrm{CH}_{3} \mathrm{CO} \bullet+\mathrm{CH}_{3} \mathrm{OCH}_{3}$ & 0.4 & 22.0 \\
\hline & \multicolumn{2}{|c|}{ average of 23 reactions: } & $20.1 \pm 4.0$ \\
\hline & \multicolumn{2}{|c|}{ experiment (ATcT): } & $21.53 \pm 0.34$ \\
\hline
\end{tabular}

${ }^{a}$ Experimental enthalpies of formation of all species used in isodesmic reactions are given in Table $\mathrm{S} 1$. These values were taken from the ATcT except for the blue marked species. ${ }^{b}$ The values of enthalpies of reaction $\left(\Delta_{\mathrm{r}} H_{298}^{\circ}\right)$ are given together with enthalpies of formation $\left(\Delta_{\mathrm{f}} H_{298}^{\circ}\right)$ for each isodesmic reaction. 